\title{
ARTICLE OPEN Spatial transcriptomic survey of human embryonic cerebral cortex by single-cell RNA-seq analysis
}

\author{
Xiaoying Fan ${ }^{1,2}$, Ji Dong ${ }^{1,2}$, Suijuan Zhong ${ }^{3,4,5}$, Yuan Wei ${ }^{1,6}$, Qian $\mathrm{Wu}^{3,4,5}$, Liying Yan ${ }^{1,6}$, Jun Yong ${ }^{1,6}$, Le Sun ${ }^{3,4,5}$, Xiaoye Wang ${ }^{1,6}$, \\ Yangyu Zhao ${ }^{1,6}$, Wei Wang ${ }^{1,6}$, Jie Yan ${ }^{1,6}$, Xiaoqun Wang ${ }^{3,4,5,7}$, Jie Qiao (iD) $1,6,8,9$ and Fuchou Tang (iD) ${ }^{1,2,9}$
}

The cellular complexity of human brain development has been intensively investigated, although a regional characterization of the entire human cerebral cortex based on single-cell transcriptome analysis has not been reported. Here, we performed RNA-seq on over 4,000 individual cells from 22 brain regions of human mid-gestation embryos. We identified 29 cell sub-clusters, which showed different proportions in each region and the pons showed especially high percentage of astrocytes. Embryonic neurons were not as diverse as adult neurons, although they possessed important features of their destinies in adults. Neuron development was unsynchronized in the cerebral cortex, as dorsal regions appeared to be more mature than ventral regions at this stage. Regionspecific genes were comprehensively identified in each neuronal sub-cluster, and a large proportion of these genes were neural disease related. Our results present a systematic landscape of the regionalized gene expression and neuron maturation of the human cerebral cortex.

Cell Research (2018) 28:730-745; https://doi.org/10.1038/s41422-018-0053-3

\section{INTRODUCTION}

The adult brain of vertebrate animals has extensive capabilities due to its astonishing cell type diversity ${ }^{1,2}$ and precise arrangement of regional structures, ${ }^{3}$ especially in the cerebral cortex as it is the most evolved organ with the most complex functions in human. The cerebral cortex contains convoluted, layered gray matter that is only $2-3 \mathrm{~mm}$ thick in human but with several hundred square centimetres of surface area. ${ }^{4}$ Neurons residing in the gray matter are the basic unit in the system and possess outgoing axons that club together to form the white matter of the cerebral cortex. Neurons located in different cortical layers and regions project to their specific destinations where they can receive and release signals by transmitting neurotransmitters to feel and control..$^{5-7}$ Previous classifications for neurons were mainly based on their morphological, chemical, and electrical properties. As these properties are controlled genetically, neuron sub-cluster classifications have been defined by distinct molecular characteristics in recent studies. ${ }^{8-12}$

The enormous diversity of neurons with precise framework comes from genetically committed neural stem cell (NSC) and progenitor pools. ${ }^{13,14}$ Apart from the diverse neurons, progenitor pools produce more abundant glial cells including astrocytes and oligodendrocytes. ${ }^{2}$ These glial cells do not transmit signals like neurons, but they constitute the environment to chaperon the neurons and shape the neuronal network, ${ }^{14,15}$ and their dysfunction is associated with many neural system diseases. ${ }^{16-18}$ Although we have known that the neuronal and glial lineages share the same origin, the genetic determinants diversifying the neural progenitors into neuronal or glial specification are still not fully understood.

As the major architecture of the adult brain is almost established at the embryonic stage, dissecting the cell complexity and specific regional features of the developing cortex is a promising strategy for studying the functional specialization of the cerebral cortex. Previous studies, which have analyzed temporal and spatial neural development in rodent, human, and nonhuman primate brains, and have uncovered specific regional and temporal molecular characteristics of brain development, were almost based on bulk RNA-seq analysis. ${ }^{19-24}$ The molecular profiles of each structure can be unveiled by analyzing microdissected cortical tissues. However, such assessments are far from revealing the detailed mechanisms of cerebral cortex organization, as dissected structures are still composed of multiple cell types.

Single-cell transcriptome analysis may provide more precise information according to current progress, especially on cell type diversities, ${ }^{8-11,25-31}$ but barely approach the regional information to reveal the transcriptional landscape of the entire human

\footnotetext{
13eijing Advanced Innovation Center for Genomics, Department of Obstetrics and Gynecology, College of Life Sciences, Third Hospital, Peking University, Beijing 100871, China;

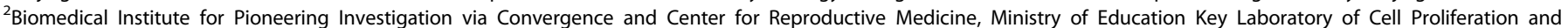

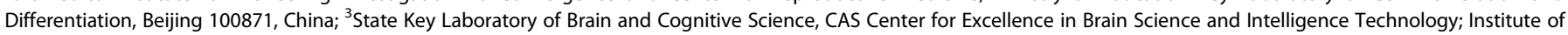

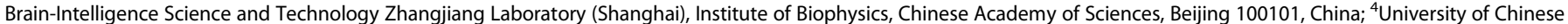

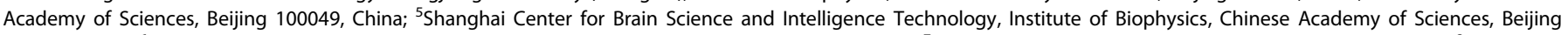

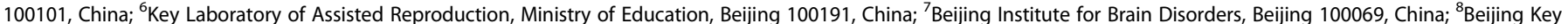

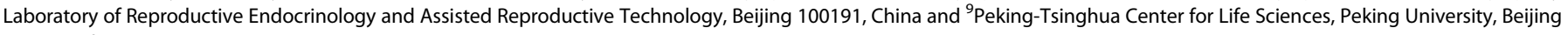
100871, China

Correspondence: Xiaoqun Wang (xiaoqunwang@ibp.ac.cn) or Jie Qiao (jie.qiao@263.net) or Fuchou Tang (tangfuchou@pku.edu.cn)

These authors contributed equally: Xiaoying Fan, Ji Dong, Suijuan Zhong, and Yuan Wei
}

Received: 1 February 2018 Revised: 9 April 2018 Accepted: 2 May 2018

Published online: 4 June 2018 
embryonic cerebral cortex at single-cell resolution. In this study, we collected single cells for transcriptome analysis from different regions of the entire human cortex at 22 and 23 weeks postconception ( $22 \mathrm{~W}$ and $23 \mathrm{~W}$ ) and supplied the first data source to lay the ground for understanding the cell type constitution and molecular differences of regional development in the whole human cerebral cortex at the mid-gestational stage.

\section{RESULTS}

Global clustering and identification of the single cells

To detect the molecular distributions of 20 major anatomical cortical regions together with the medulla and the pons, we picked single cells as summarized in Supplementary information, Table S1. A total of 4,213 single cells from the cerebral cortex of a $22 \mathrm{~W}$ embryo and two $23 \mathrm{~W}$ embryos were analyzed. An average of $1.3 \times 10^{6}$ mappable reads were generated for each cell, and on average, 4,318 genes were detected in each individual cell. We performed the t-distributed stochastic neighbor embedding ( $t$ SNE) analysis to explore the diversity of all these cells. The even mixture of cells from different embryos in each cluster on the tSNE plot reflected negligible individual variance or a batch effect for each cell type (Supplementary information, Figure S1a). Based on the global expression patterns, all cells were classified into three major groups, namely, neurons, glial cells and non-neural cells (Fig. 1a).

Further, each major group was divided using a random forest algorithm and we obtained eight major cell types, including inhibitory neurons, excitatory neurons, Cajal-Retzius cells, glial cells, microglia, endothelial cells, immune cells, and blood cells (Fig. 1a and Supplementary information, Table S2). Classical markers were specifically detected in the corresponding clusters of cells (Supplementary information, Figure S1b). In total, 1,672 differentially expressed genes (DEGs) were obtained among these major cell types using Seurat analysis. Gene ontology (GO) analysis also showed that each type of cell specifically expressed genes involved in the corresponding biological processes, as expected (Fig. $1 \mathrm{~b}$ and Supplementary information, Table S3).

Neuronal cells (inhibitory neurons, excitatory neurons and CajalRetzius cells) accounted for $66 \%$ of the cells in the $22 / 23 \mathrm{~W}$ cerebral cortex, whereas glial cells (which could be further divided into NSCs, oligodendrocyte progenitor cells (OPCs), oligodendrocytes, and astrocytes) only accounted for $8 \%$ of the cells (Fig. 1c and Supplementary information, Figure S1c). This cellular distribution is markedly different from that found in the gray matter of the adult cerebral cortex, where glial cells are thought to outnumber neurons by 1.5 times. ${ }^{32}$ However, co-immunostaining for NEUROD1 and GFAP validated the much higher abundance of excitatory neurons than astrocytes in different cortical regions at this embryonic stage (Supplementary information, Figure S1d). Thus, we deduced that the preferential expansion of glial cells over neurons in the cerebral cortex occurs after 23 weeks postfertilization in humans. Very different from the cortical regions, regions in the inferior surface such as the insular gyris (IG) and medulla contain more glial cells (Supplementary information, Figure S1e). More microglia were observed in the medulla than in the IG region, while these microglia in the medulla seemed less developed than those in the IG regions as the former are ameboid whereas the latter start to develop ramified form (Supplementary information, Figure S1e). This indicates that microglia development was unsynchronized in different brain regions. The vast majority of the cells in the cortex were in a quiescent/post-mitotic state at this embryonic stage, except that $\sim 40 \%$ of the NSCs were still actively dividing (Supplementary information, Figure S1f).

Neuron sub-clusters in the human embryonic cortex

To further reveal the subpopulations of each major cell type, we performed a more detailed analysis of each cell type with the
Single-cell RNA-seq of human embryonic cerebral cortex $X$. Fan et al.

random forest algorithm. A total of 968 inhibitory neurons were 731 further divided into 8 sub-clusters, with each sub-cluster characterized by unique marker genes such as neural peptides $(N X P H 1, S S T)$, enzymes (PAM), important calcium-binding proteins (CALB2, CAMLG), transcription factors (TFs) ( $L H X 6)$, and non-coding RNAs regulating GABAergic cell fate (DLX6-AS1) (Fig. 2a). Inhibitory neuronal subgroups 1-4 highly expressed LHX6 (Fig. 2b); these subgroups are thought to be generated in the medial ganglionic eminence (MGE), a major source of the GABAergic population in the cerebral cortex. ${ }^{33}$ These cells expressed LHX6 together with NXPH1 and PAM, and accounted for $50.9 \%$ of all the inhibitory neurons in the cortex. Furthermore, $24.9 \%$ of the $\mathrm{LHX6}^{+}$ progenitors developed into a somatostatin (SST)-expressing population (subgroup In_2). We identified a BOD $1 L 1^{+}$subgroup among the inhibitory neurons (Fig. 2b,c). This subgroup of cells did not express POLR2E, which encodes a subunit of RNA polymerase II. The other $49.1 \%$ of the inhibitory neurons (subgroups 5-8) were CALB2 positive. These cells expressed both DLX2 and NR2F2 (encoding COUP-TFII) (Fig. 2c), indicating that they may originate from the caudal ganglionic eminence (CGE) and then migrate into the cortex. ${ }^{34}$ By calculating the proportions of $\mathrm{LHX6}^{+}$and $\mathrm{CALB2}{ }^{+}$inhibitory neurons in each cortical region, we found that $\mathrm{LHXG}^{+}$neurons are relatively enriched in the superior part of the cerebral cortex, whereas the $C A L B 2^{+}$neurons are enriched in the inferior regions (Fig. 2c, d).

The 1,625 excitatory neurons were further subdivided into 4 groups, and groups 1 and 2 showed higher expression of more immature genes, such as EIF1B, MEIS2, and LINC01158 ${ }^{35}$ (Fig. 2b). The ortholog of LINC01158 in mouse, Pantr1, regulates the differentiation of neuronal progenitors, whereas its paralog Pantr2 regulates the expression of Pou3f3 and maintains the proliferation of progenitor cells in the developing cortex. ${ }^{36,37}$ The expression pattern of LINC01158 indicated a different role in humans compared with that in rodents. The other two subgroups were more mature, as these groups highly expressed SYBU and CELF4. FOXP1, a TF important for medium spiny projection neurons, was also detected in the excitatory neuron subgroups 3 and 4 in the cerebral cortex. ${ }^{38}$ Based on a pseudotime analysis, all the excitatory neurons were arranged according to a developmental course. Subgroup 1 (Ex_1) to subgroup 4 (Ex_4) cells ranked sequentially from earlier to later in the pseudotime course, as expected (Fig. 2e, left). Genes down-regulated during the differentiation course of the excitatory neurons were mainly associated with negative regulation of RNA polymerase II transcription and cytokine production. These genes were more enriched in germinal structures (VZ, ISVZ, and OSVZ), as reported by Miller et al. $^{20}$ (Fig. $2 \mathrm{e}$ and Supplementary information, Figure S2a). In contrast, the positively regulated genes were enriched in synaptic functions, such as membrane depolarization during action potentials, and showed higher expression levels in the upper structures of the cortex (IZ to MZ). These genes were enriched in neuron-related functions, such as membrane depolarization during action potential and signal release (Fig. $2 \mathrm{e}$ and Supplementary information, Figure S2a). These findings suggest that the molecular mechanism underlying the excitatory neuron differentiation could be revealed by scRNA-seq.

Since subgroups Ex_1 to Ex_4 showed a developmental relationship based on pseudotime analysis, we picked the TFs that potentially regulated the maturation of excitatory neurons from DEGs among these four groups. Four TFs (ZGLP1, HIC2, $P O U 2 F 2$, and $F O X K 1)$ potentially positively regulated the maturation of excitatory neurons and another four TFs (MEIS2, ZBTB20, $N F I A$, and $Z F H X 4)$ potentially negatively regulated the maturation of excitatory neurons in the developing human cortex, respectively (Fig. 3a). We further analyzed the regulation network of these eight TFs (Fig. 3b and Supplementary information, Table S4) by analyzing the genes co-expressed with the TFs and extracted the top 1,000 links showing positive correlation with each TF. We 
X. Fan et al.

a
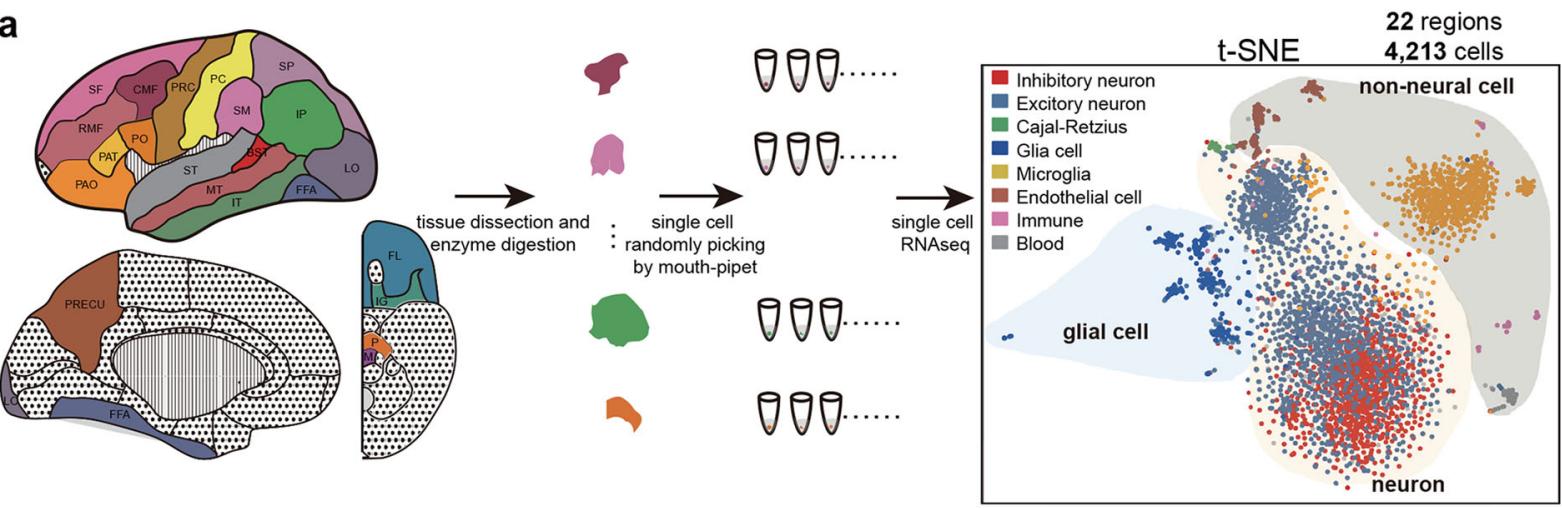

b

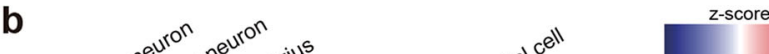

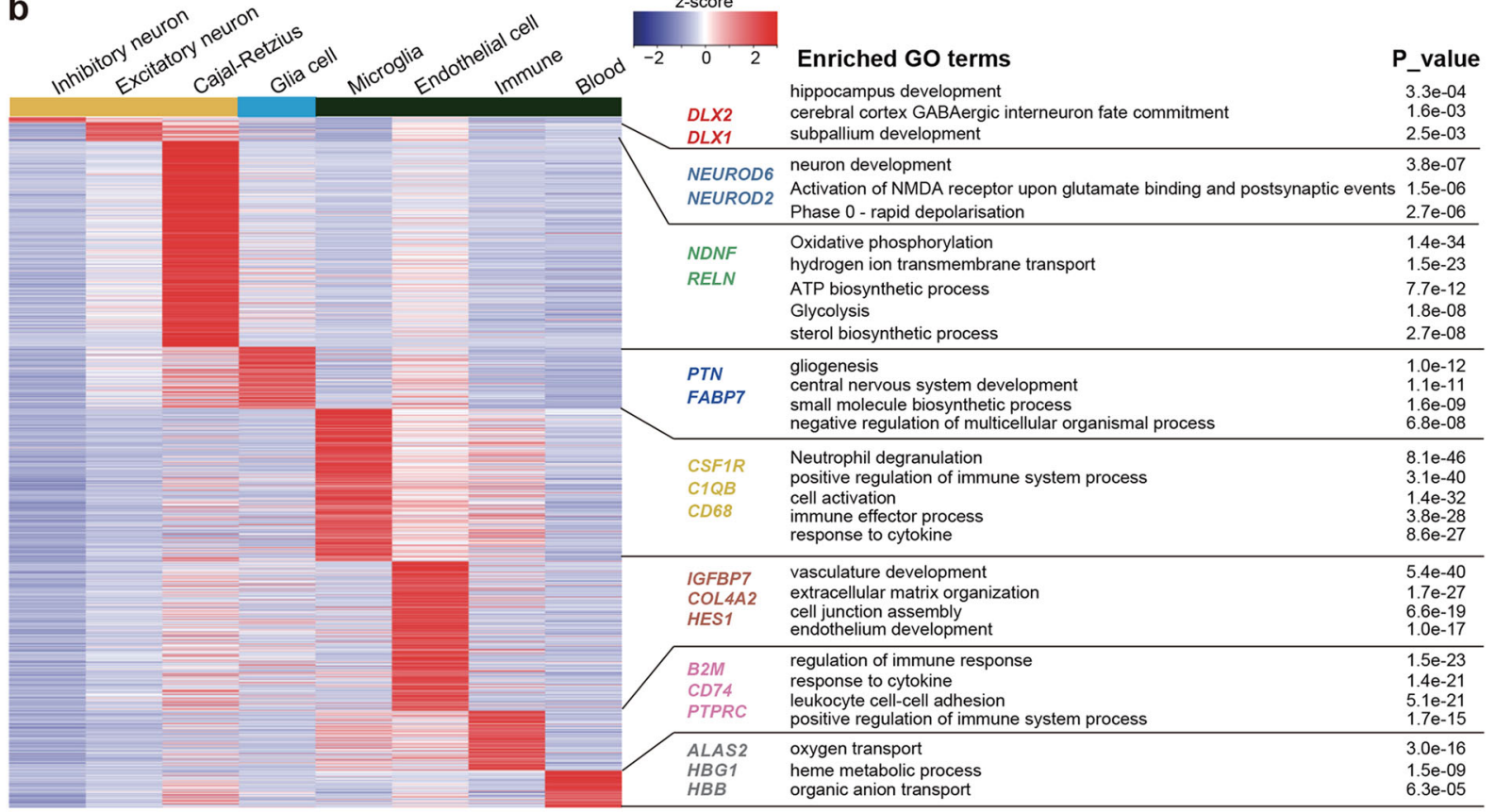

C
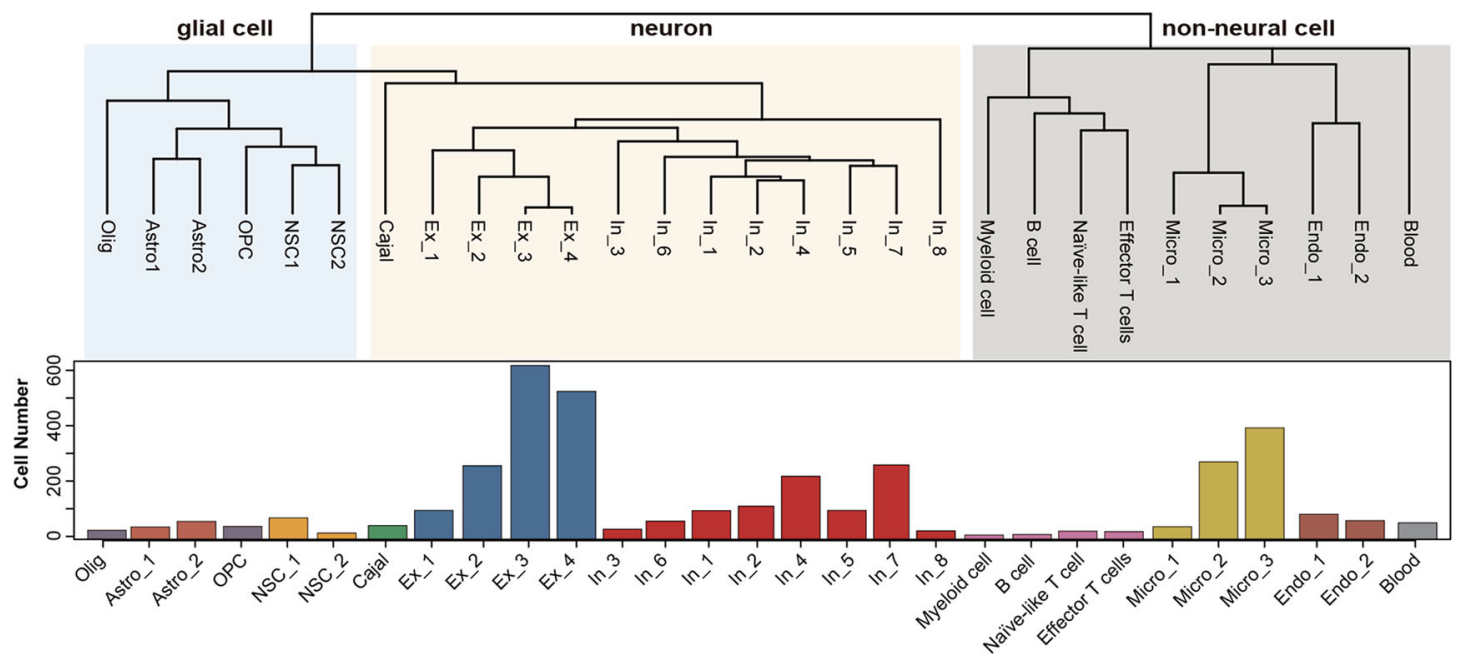

Fig. 1 Cell type classification in human embryonic cerebral cortex. a The schematic diagram displaying the dissection of embryonic brain and how we obtained the single cell transcriptome data in 22 regions (for the abbreviations, see Supplementary information, Table S1). t-SNE showed the cell types identified with all the single cells and the shadows mark different general cell types: the blue shade indicates glial cell, the yellow shows neuron and the gray shows non-neural cell that is not supposed to be produced by neural stem cell. b Heatmap displaying the DEGs that were cell type specific in our analysis. The enriched biological processes for each gene group were shown in the right. Classical cell type marker genes were labeled. c Dendrogram showing the relationships of all the 29 sub-clusters and the histogram displaying the cell number in each sub-cluster 
a

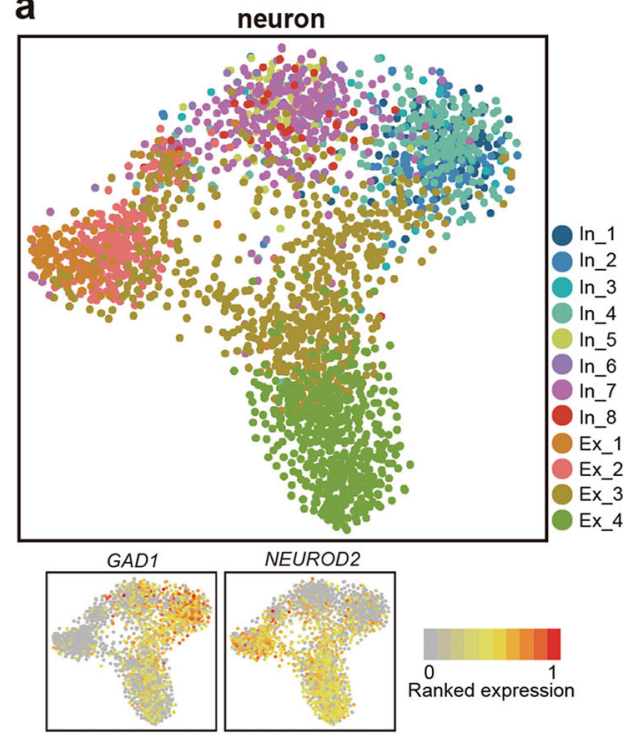

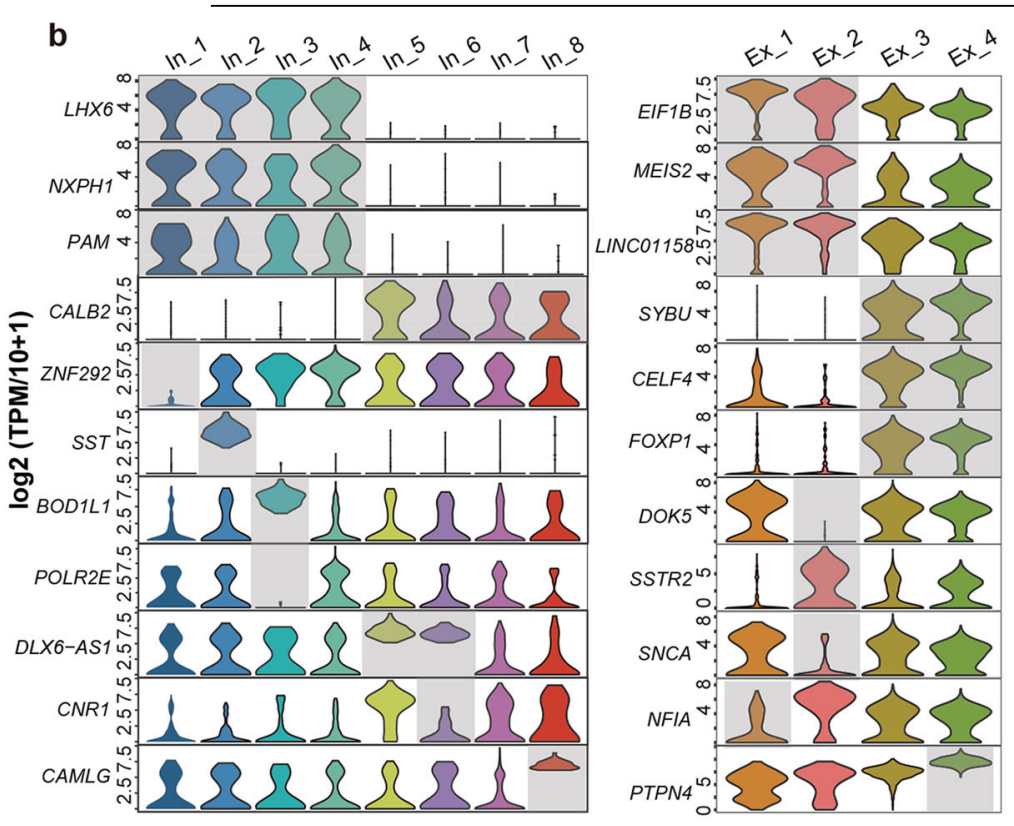

C

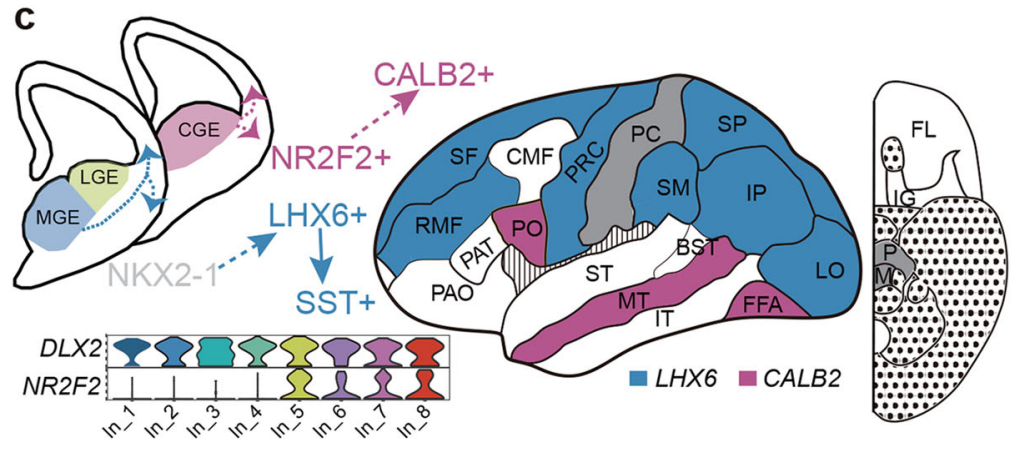

d

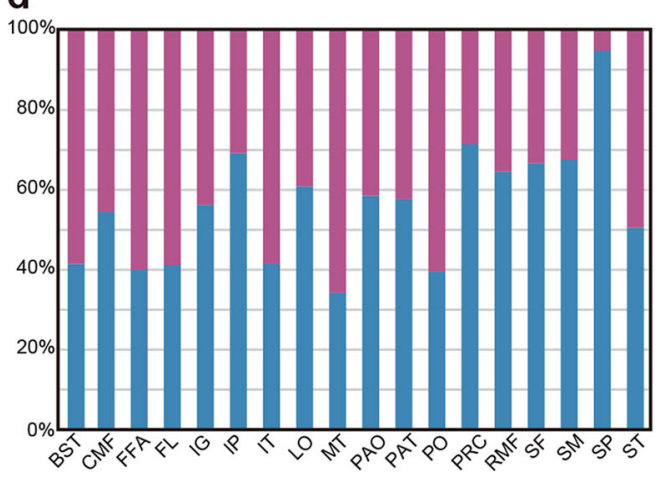

e

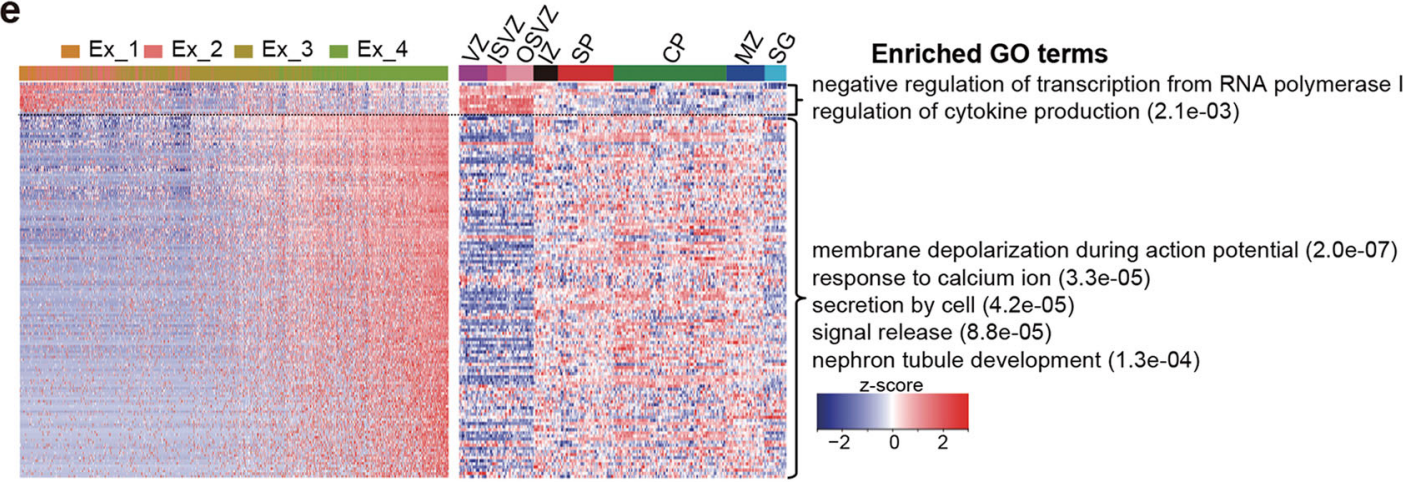

Fig. 2 Neuron cell sub-clusters in human embryonic cerebral cortex. a t-SNE map showing the subtypes of all inhibitory and excitatory neurons. The inhibitory neuron could be subdivided into eight clusters, and all of those are GAD1 positive. The excitatory neurons are NEUROD2 positive and could be subdivided into four clusters. In inhibitory neuron, Ex excitatory neuron. b Violin plot showing the DEGs of subgroups with inhibitory neuron (left) and exicitatory neuron (right), respectively. c Schematic diagram describing where different types of inhibitory neurons are generated and how they migrated. Violin plots show expression levels of interneuron progenitor genes $D L X 2$ and NR2F2 in each subgroups. The cortex landscape shows the dominant inhibitory neuron types in each region. Blue indicates that over $60 \%$ inhibitory neurons in the corresponding region are in $L H X 6$ subtype and the purple indicates that over $60 \%$ inhibitory neurons are in CALB2 subtype. White indicates that both subtypes of inhibitory neurons make up $40 \%-60 \%$ of the sum. Regions of gray color are detected with $<15$ inhibitory neurons. $\mathbf{d}$ The accurate percentages of inhibitory neurons belonging to LHX6 and CALB2 subgroups in regions colored with blue, purple, and white. e Left, heatmap showing the genes positively and negatively regulating excitatory neuron maturation, respectively. The color bars at the top represent cells from different clusters, which are arranged in a pseudotime order from immature to mature neurons. Middle, expression of putative excitatory neuron maturation regulating genes in the structures studied by Miller et al. ${ }^{20}$ (sample 12566). Right, enriched biological processes for down-regulated and up-regulated genes in excitatory neuron maturation. VZ ventricluar zone, ISVZ inner subventricular zone, OSVZ outer subventricular zone, IZ intemediate zone, SP subplate zone, CP cortical plate, MZ marginal zone, SG subpial granular zone 


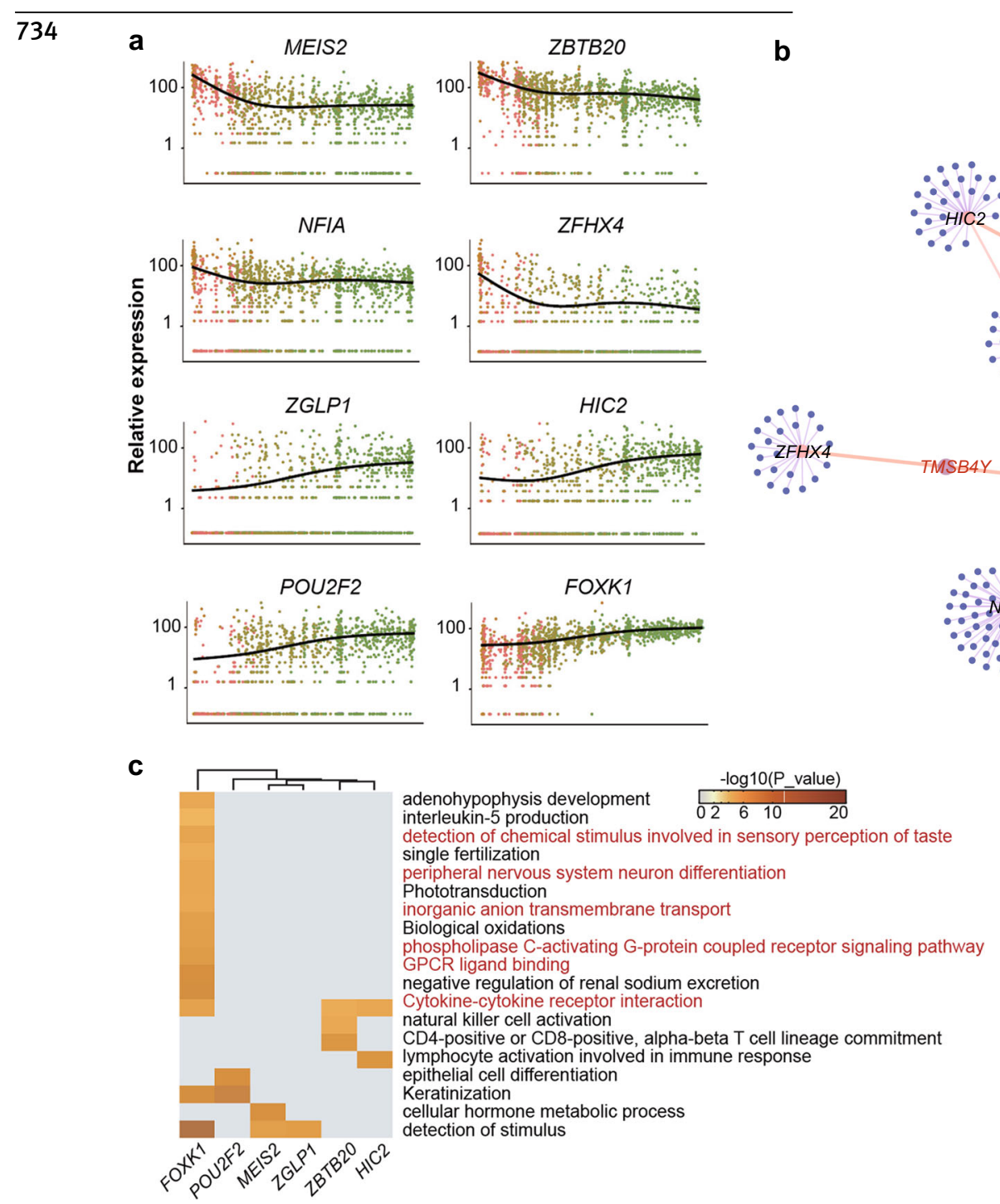

Fig. 3 Regulation network of the transcription factors in excitatory neuron differentiation. a The expression changes of TFs negatively (top row) and positively (bottom row) regulating the maturation of excitatory neurons along the pseudotime. $\mathbf{b}$ The transcription networks of the TFs regulating excitatory neuron maturation. Top 1,000 target genes for each TF were listed in Supplementary information, Table S4. c Enrichment analysis on target genes for each TF shown in $\mathbf{b}$ and $\mathbf{c}$. No enriched terms for NFIA and ZFHX4. Terms colored in red are known pathways quite related to neural development

found that FOXK1 was a candidate core TF regulating the maturation of excitatory neurons since it showed the strongest co-regulation patterns with potential target genes. Analysis of biological process enrichment in the target genes of each TF further suggested that FOXK1 participated in multiple processes of neuronal maturation (Fig. 3c).

Comparison of human embryonic neurons to adult neurons in the cortex

Lake et al. revealed the molecular signatures of neurons in different cortical regions of the human adult brain. ${ }^{26}$ We wondered whether these signatures were already formed in the mid-gestational embryonic cortex. First, we analyzed the expression levels of excitatory and inhibitory neuron sub-cluster markers identified in the adult cortex (Fig. 4a). Approximately half of the excitatory sub-cluster markers were expressed in the embryonic excitatory neurons; these markers could be used to discern only the Ex_1/2 from the Ex_3/4 subgroups. Few of the inhibitory subcluster markers were detected in the embryonic inhibitory neurons, perhaps because both the excitatory and inhibitory neurons at this embryonic stage were not fully developed with functional projections, and most of these neurons were still progenitors during migration. Further analysis of the expression levels of the layer markers also showed no patterns of these adult neuron markers on the embryonic neuronal sub-clusters for either excitatory or inhibitory neurons (Fig. 4b). Only the more mature sub-clusters of excitatory neurons, Ex_3 and Ex_4, showed a broad distribution across layer $6 \mathrm{~b}$ to layer 2 and these cells expressed markers of multiple layers (Supplementary information, Figure $\mathrm{S} 2 \mathrm{~b})$. We suspected that these migrating neurons express multiple layer signatures at $23 \mathrm{~W}$, and later, when they have arrived at their final destination, they will mature and express marker genes specific for the layer that they migrate into and develop full projections.

We noticed that the expression level of upper layer marker CUX $2^{39,40}$ could clearly specify the four sub-clusters of excitatory neurons and the more mature neurons showed higher expression of CUX2 (Fig. 4b). Thus, CUX2 could be used to evaluate the maturation degree of each region in the whole cerebral cortex. According to the $\mathrm{CUX2}^{+}$cell ratio in each region, we found that 
a

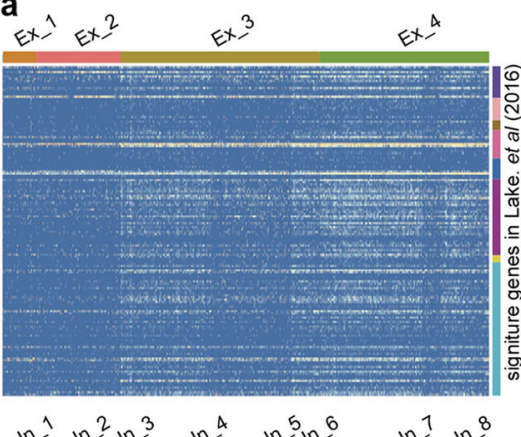

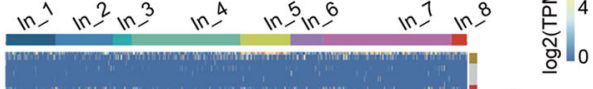

C

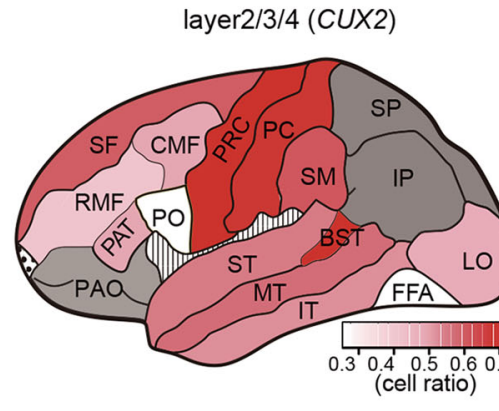

b

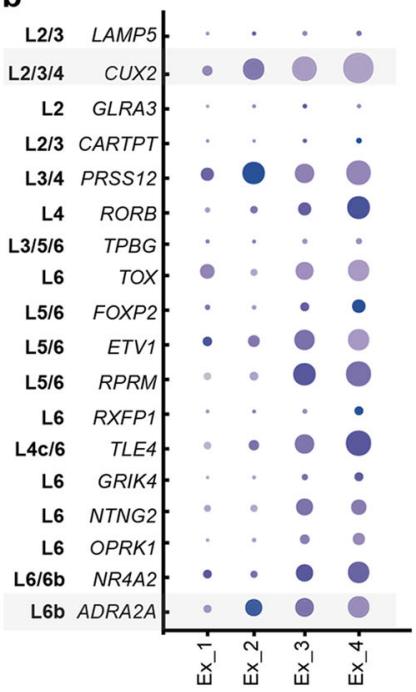

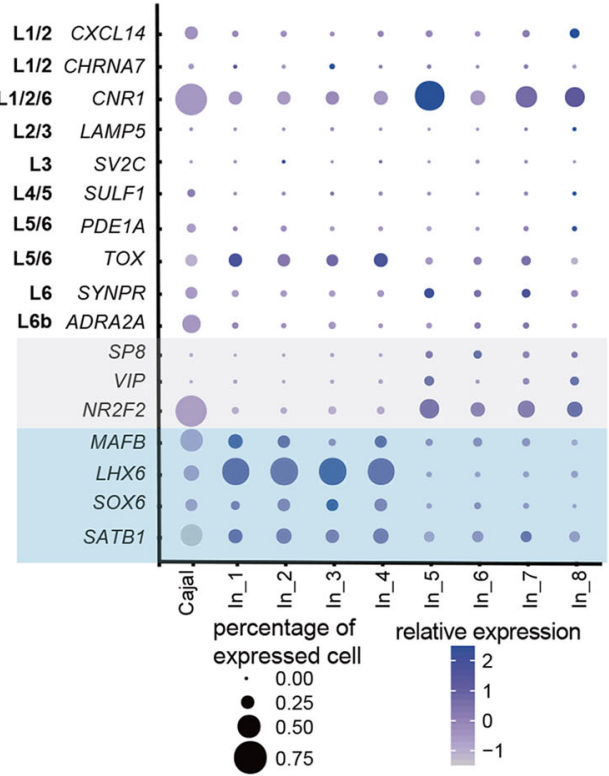

e
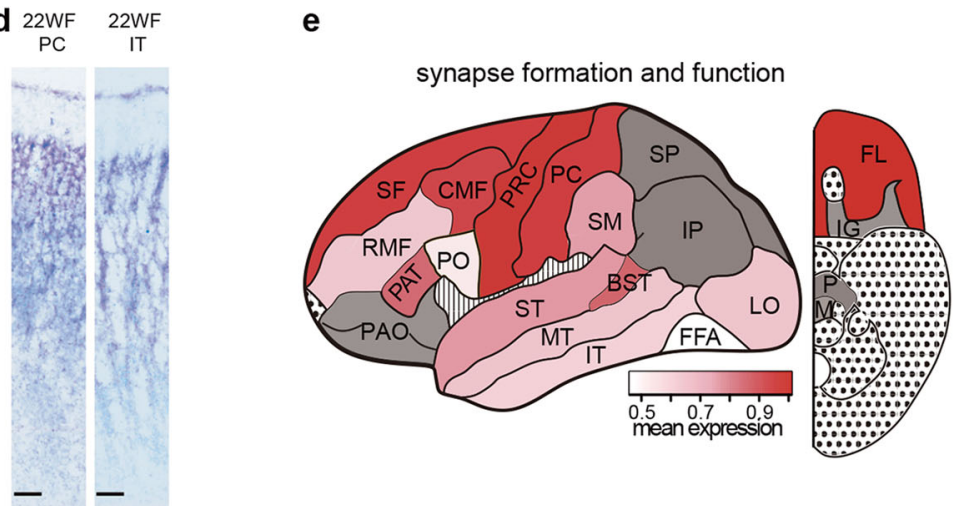

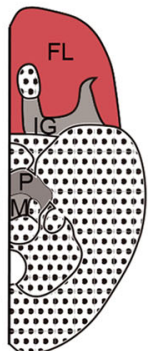

Fig. 4 Maturation degree of embryonic neurons is different from the adult neurons. a Heatmap showing the expression of marker genes identified in the adult neuron subtypes. $\mathbf{b}$ Expression of layer markers in different sub-clusters of excitatory neuron (left) and inhibitory neuron (right). c Landscape showing the maturity level of each region in the cortex measured by CUX2-positive excitatory neuron ratio. Regions of gray color are ruled out as they are detected with $<50$ neurons. $\mathbf{d}$ In situ hybridization of CUX2 in PC and IT regions of a 22 WF embryo. Scale bar, $100 \mu \mathrm{m}$. e Landscape showing the maturity level of each region in the cortex measured by expression level of synapse formation and function related genes

the neurons in the pre-central cortex (PRC, also known as the primary somatomotor cortex) and the post-central cortex (PC, also known as the primary somatosensory cortex) were more mature than the neurons in other cortical regions (Fig. 4c). RNA in situ hybridization of CUX2 was performed for the PC and the inferior temporal cortex (IT) regions, which indeed confirmed more CUX2 ${ }^{+}$ cells in the PC region (Fig. 4d). Similar mature patterns were revealed when we analyzed the expression levels of synapse formation and function related genes as measures of neuron maturity in each cortical region (Fig. 4e).

To obtain further insight into the differences between embryonic and adult cortex neurons, we compared our singlecell data with those of the adult neurons by principal component analysis (PCA) after removing batch effect (Fig. 5a). The findings showed that the heterogeneity of the excitatory neurons at midgestation stage was already comparable to that of the adult brain. Both PC1 and PC2 reflected the differences between immature and mature embryonic neurons, and the enriched terms were also associated with projection development and synaptic plasticity (Fig. 5b). The results for inhibitory neurons were very different. PC1 genes clearly separated the embryonic inhibitory neurons from the adult neurons (Fig. 5c). The adult inhibitory neurons highly expressed PC1 positive direction genes, which were enriched in neuron signaling genes, whereas the embryonic inhibitory neurons specifically expressed PC1 negative direction genes, which were associated with cellular metabolism. The PC3 and PC4 genes mainly reflected the differences among the subgroups of adult inhibitory neurons, whereas the embryonic inhibitory neurons exhibited no significant differences on these two axes (Fig. 5d). We inferred that this difference might exist because the mid-gestation inhibitory neurons were still immature and had not differentiated into subtypes comparable to those in the adult cortex. Since the $\mathrm{SST}^{+}$inhibitory subgroup is classified as a subcluster at this stage, we wondered whether this subtype of neuron matures earlier than other subtypes of inhibitory neurons. We combined our inhibitory neurons with those identified in the developing pre-frontal cortex ${ }^{26}$ and performed pseudotime analysis. Inhibitory neurons at $22-23 \mathrm{~W}$ in this study mainly located from early to the middle developing stages on the pseudotime path (Fig. 5e). The SST ${ }^{+}$neurons randomly appeared in the whole developing course of the pseudotime path, indicating that they were not more mature compared to other 

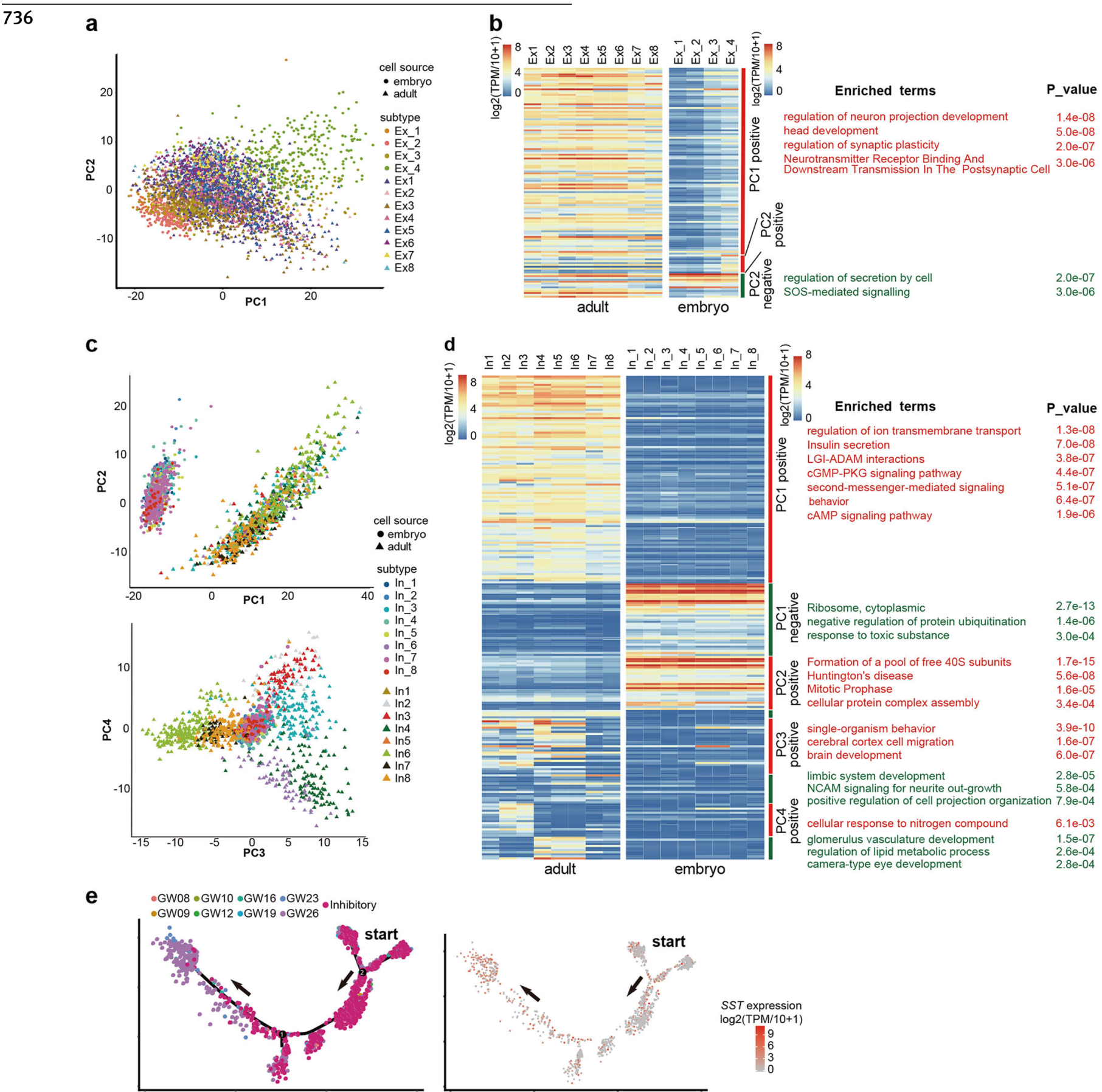

Fig. 5 Comparison of embryonic neuron sub-clusters to adult ones. a PCA plot of both excitatory neuron sub-clusters and adult ones. $\mathbf{b}$ Heatmap of expression of the top genes in the PCs corresponding to panel (a) and the enriched terms for each PC gene set. Red bar indicates the genes positively correlated in each PC and the green bar indicates the negatively correlated genes in each PC. c PCA plot of both inhibitory neuron sub-clusters and adult ones. d Heatmap of expression of the top genes in the PCs corresponding to panel (c) and the enriched terms for each PC gene set. e Monocle analysis of inhibitory neurons together with those identified in developing pre-frontal cortex. $S S T^{+}$cells show up randomly on the pseudotime

inhibitory neurons. Thus we inferred that the SST expression should not be of the same function as those in adult neurons.

To more thoroughly analyze the cell types of the human cortex, we also compared our data with the single-cell data from Pollen et al. $^{27}$ and Darmanis et al. ${ }^{11}$ We analyzed all the single-cell samples by PCA after batch effect correction using mutual nearest neighbor (MNN) algorithm in Scran package. ${ }^{41,42}$ The PC2 positive direction genes were enriched for the neuron progenitor-specific genes, especially for those at early embryonic stage (Fig. 6a). PC3 and PC4 clearly separated the adult cells from the embryonic cells, except for the Cajal-Retzius cells identified in our study, which mixed well with the adult cells. Thus, Cajal-Retzius cells were probably the cells that kept the most similar expression signatures from mid-gestation stage to adult. All the cells could be clustered into 8 groups, each showing a specific gene expression pattern (Fig. 6b). According to the marker gene expression signature for each group, both the actively dividing neural stem cell group and the quiescent neural stem cell group mainly consisted of GW16, 

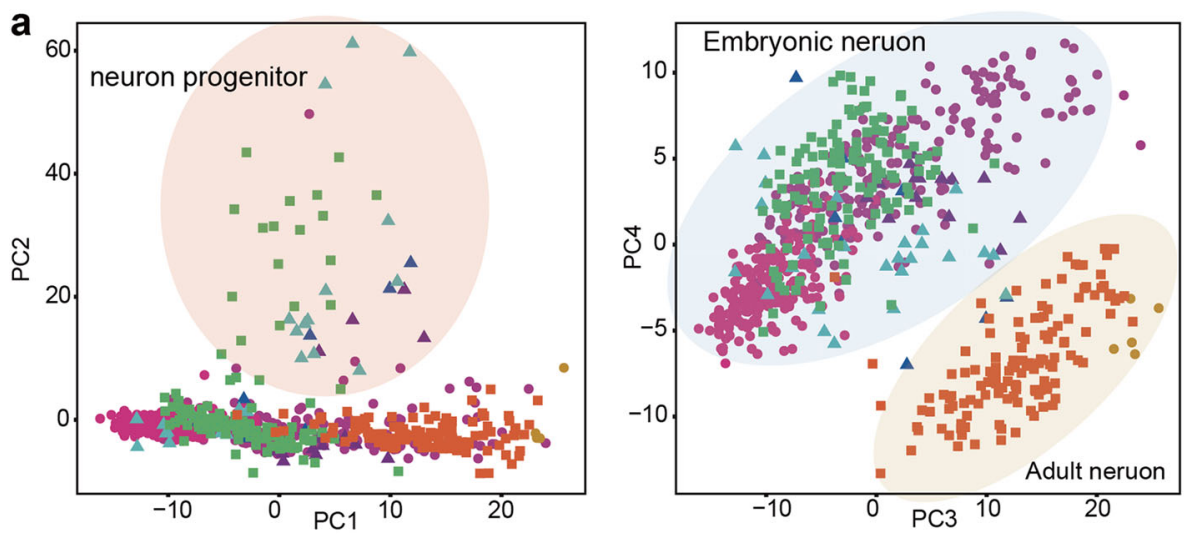

b

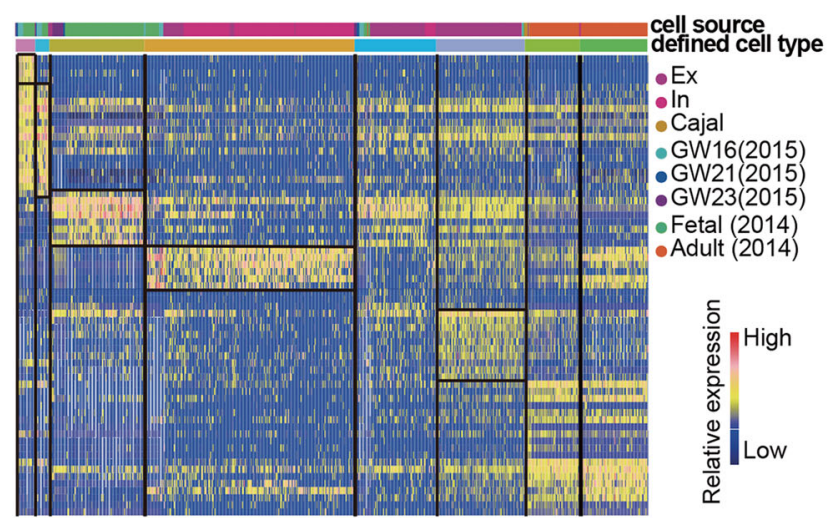

C

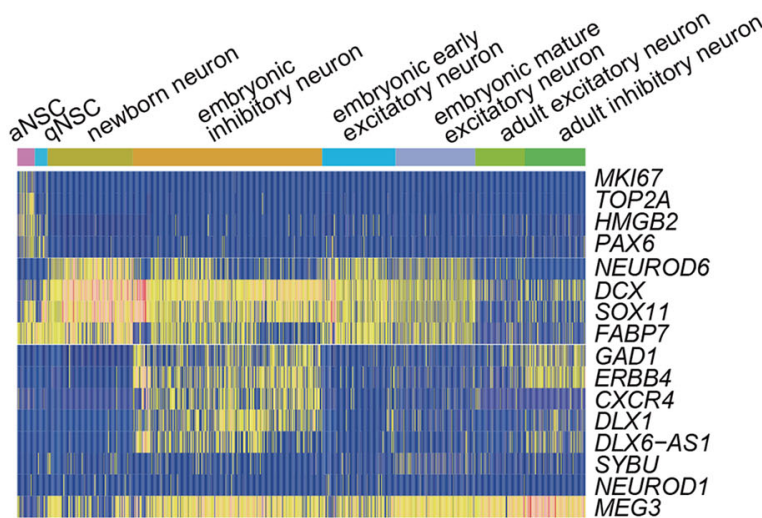

d

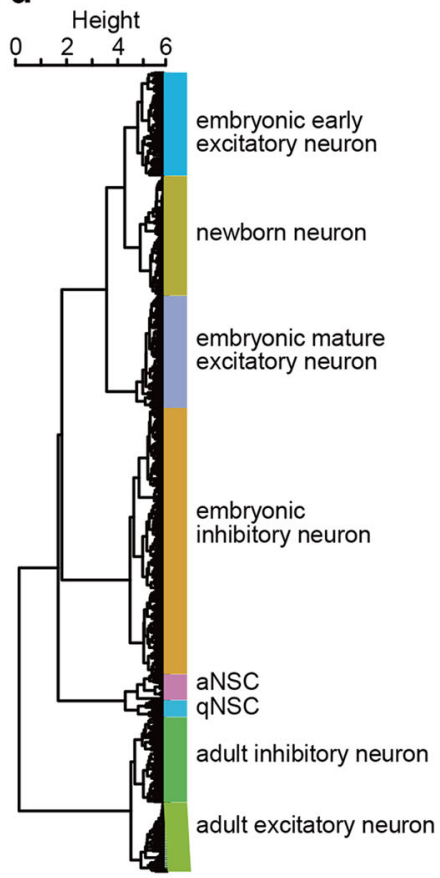

ell source

- This work

A Pollen. et al. (2014)

- Darmanis. et al. (2015)

cell type

- Ex

- In

- Cajal

- GW16(2015)

- GW21(2015)

- GW23(2015)

- Fetal(2014)

- Adult(2014)

Fig. 6 Comprehensive analysis on cell types of human cortex. a PCA of single neuronal cells from different data sets. The PC2 clearly separates progenitors from the differentiated cells, and PC3 and PC4 separate embryonic cells from the adult cells. b Heatmap showing the clustering of cells from different data sets and group-specific gene expression. c Classical marker gene expression in each identified cell group. d Unsupervised clustering of the 8 cell groups showing the distances between the cell groups

GW21, and fetal neurons (Fig. 6b, c). The differences between adult excitatory neurons and inhibitory neurons were milder than those between the embryonic excitatory neurons and inhibitory neurons. The NSCs and adult neurons were the most unique cell types. Unsupervised clustering through Pearson distance calculation among these 8 groups revealed that the longest distance for the cell groups was between the adult neurons and the NSCs (Fig. 6d).

Sub-clusters of non-neuronal cells in the human embryonic cortex Glial cells were further subdivided into six sub-clusters (Supplementary information, Figure $\mathrm{S} 3 \mathrm{a}$ and $\mathrm{b}$ ). The two sub-clusters expressing VIM were NSCS (NSC_1 and NSC_2), and NSC_2 was more active in the cell cycle. These NSCs showed a glial fate preference to differentiate into astrocytes (marked by AQP4) and OPCs (marked by PDGFRA), and further into oligodendrocytes (marked by MOBP) (Supplementary information, Figure S3b and c). The non-neural cells were further divided into three microglial sub-clusters (marked by CD68, Micro_1, Micro_2, and Micro_3), four immune sub-clusters that were further identified as $B$ cells (marked by CD52, CD79A/B), myeloid cells (marked by $L Y Z, C S T A$ ), naïve-like $T$ cells (marked by IL7R, TCF7), and effector $T$ cells (marked by NKG7, CST7). There were two endothelial sub-clusters (marked by SPARC, Endo_1 and Endo_2) and one blood cell subcluster (marked by hemoglobin genes such as HBG1) (Supplementary information, Figure S4a and b). Cell cycle analysis of these subgroups revealed that NSC_2 and Micro_1 cells were in an actively dividing state (Supplementary information, Figures S3d and S4C).

In total, 29 sub-clusters of cells were identified in the human 22-23-week embryonic cerebral cortices, and neurons accounted for the majority of them. The more mature cell types, such as 
$X$. Fan et al.

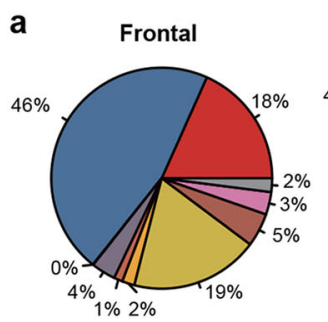

b
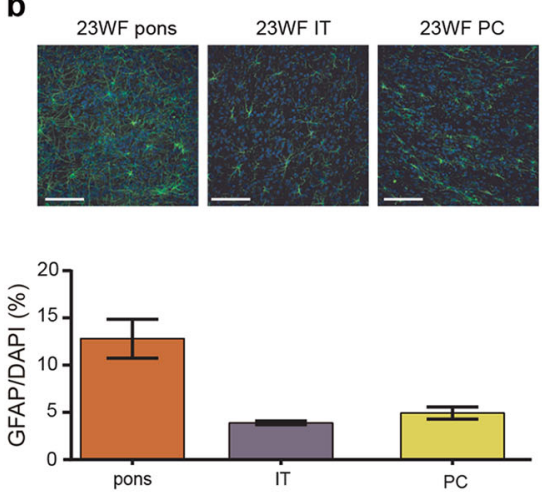

e

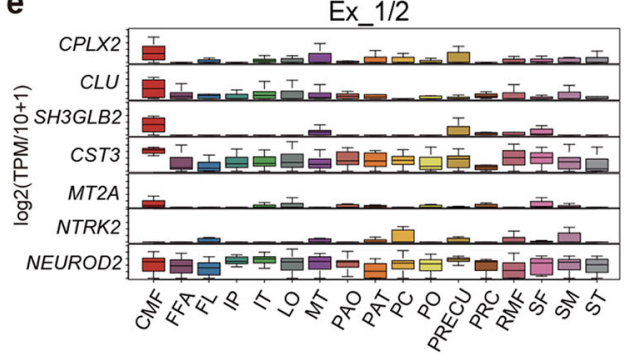

C
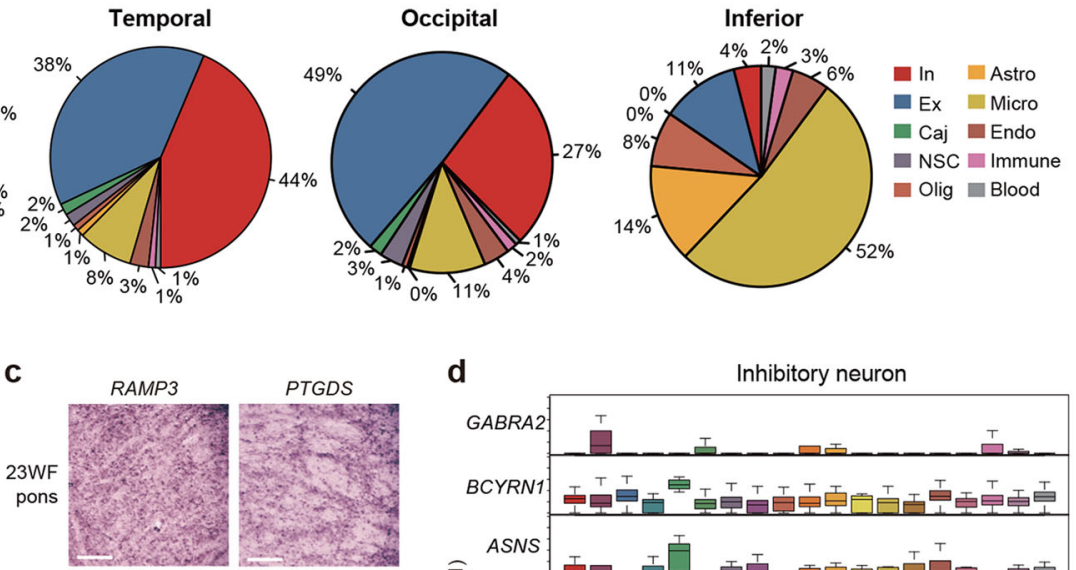

PTGDS

d

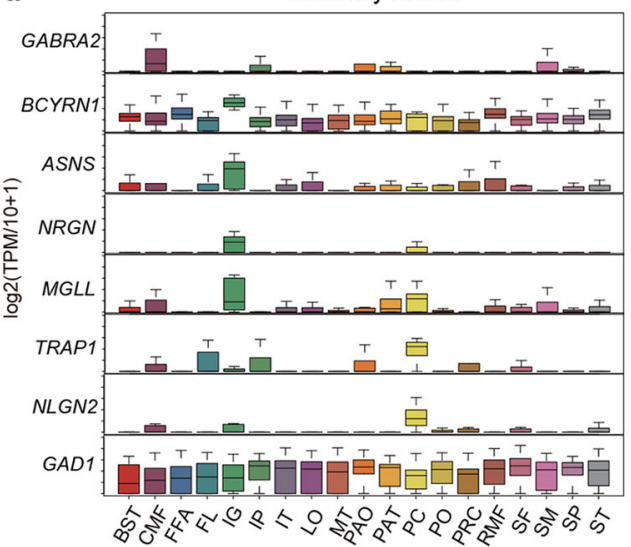

g

h

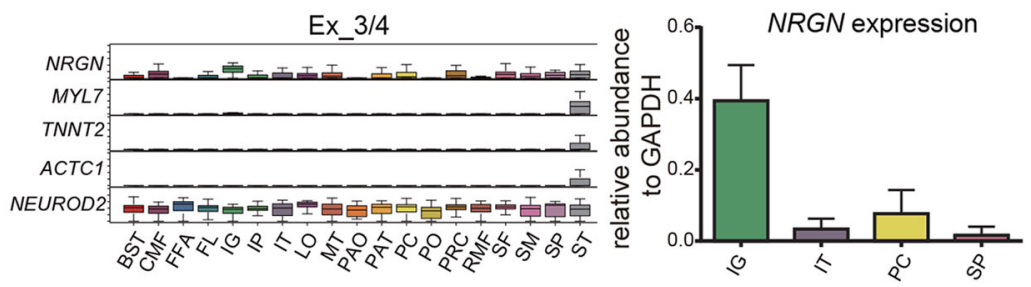

TNNT2/NEUROD2/DAPI
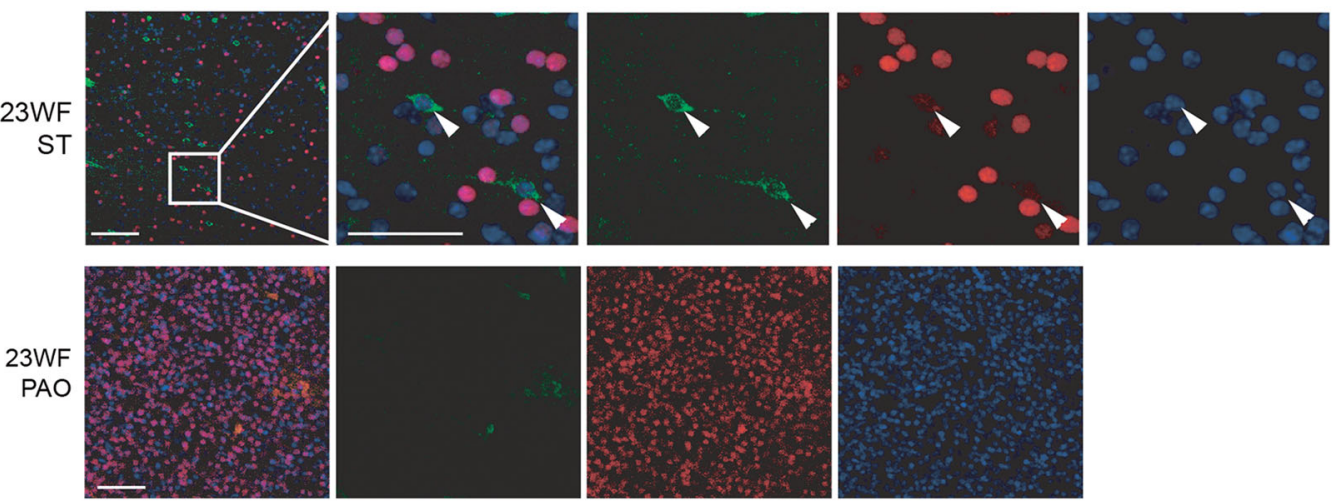

Fig. 7 Spatial differences in the developing human cortex. a Pie chart displaying the cell type constitution in the four cerebral lobes and in the inferior region of cerebral cortex. b Immunofluorescence of GFAP in the pons, PC and IT regions of a $23 \mathrm{~W}$ female sample. The statistics of GFAP $^{+}$cell ratio in each region are shown in the histogram. Scale bar, $50 \mu \mathrm{m}$. c In situ hybridization of astrocyte genes RAMP3 (Astro_1) and PTGDS (Astro_2) showing higher abundance of the two subtypes of astrocytes in pons than that in IT and PC regions. Moreover, the PC shows the lowest astrocyte density. Scale bar, $150 \mu \mathrm{m}$. d-f DEGs across all cerebral cortex regions that are detected with more than 5 inhibitory neurons (d), immature excitatory neurons (Ex_1/2, e) and mature excitatory neurons (Ex_3/4, f). GAD1 and NEUROD2 are the housekeeping control for inhibitory and excitatory neurons, respectively. g RT-qPCR of NRGN in IG, IT, PC, and SP regions. The NRGN abundance in each region was normalized by GAPDH. $\mathbf{h}$ Validation of excitatory neurons expressing myocardial protein TNNT2 in the $23 W F$ ST region by immunofluorescence. The PAO region is displayed as a negative control. TNNT2 antibody was tested in the cadiomyocytes as shown in Supplementary information, Figure S6c. Scale bar, $50 \mu \mathrm{m}$ 

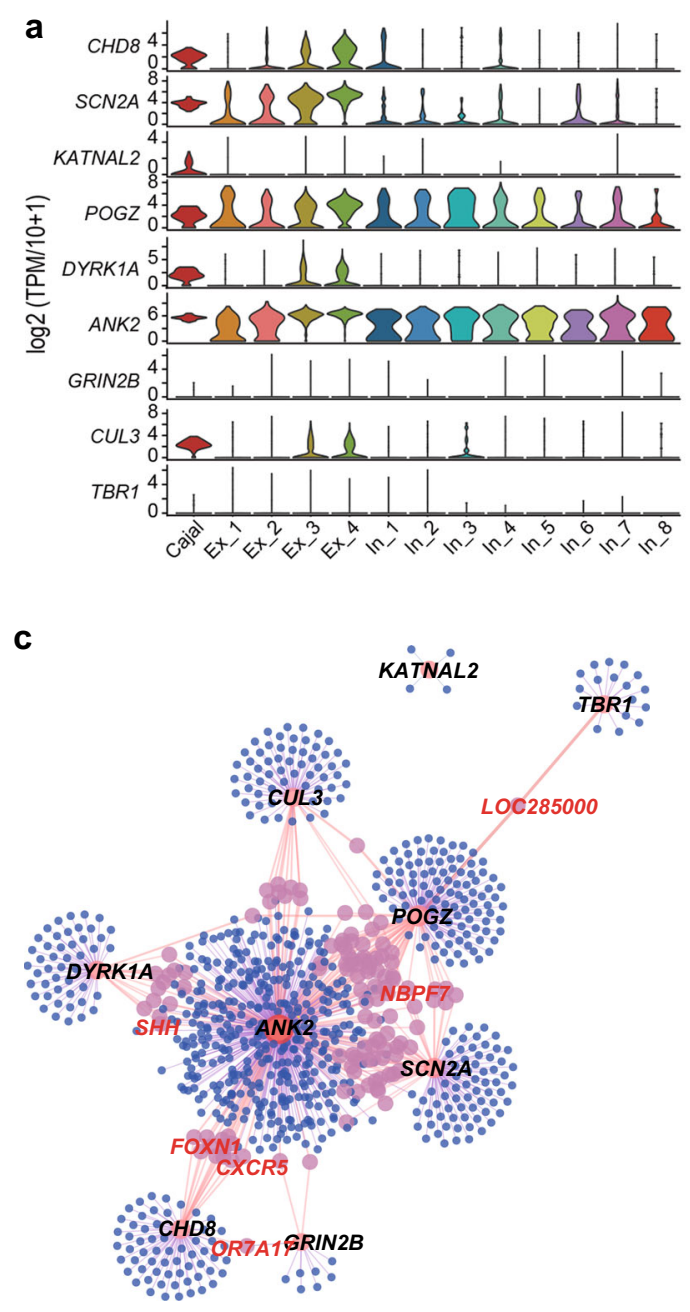

b

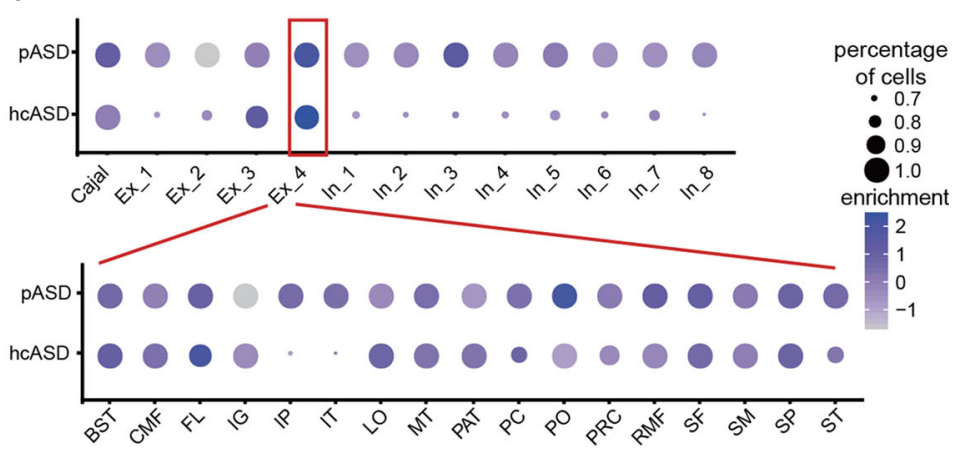

d

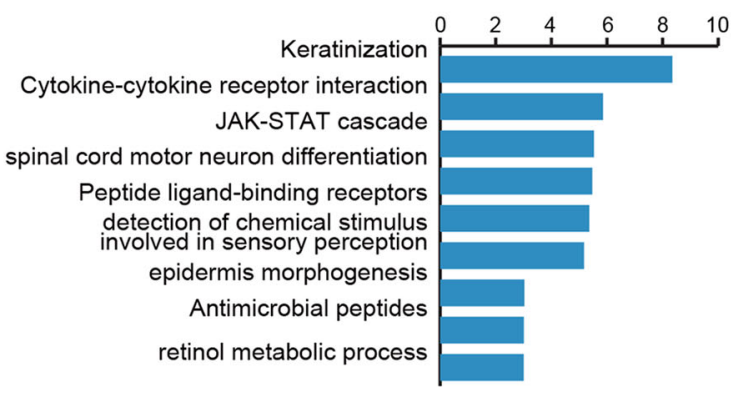

e

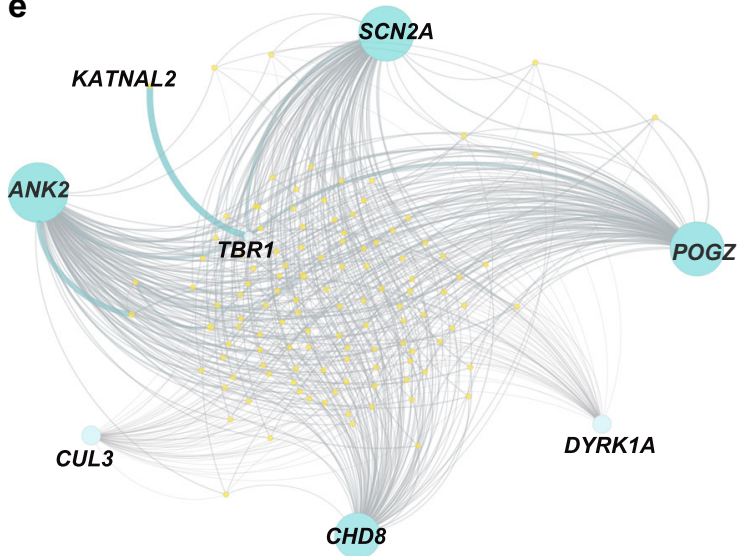

Fig. 8 Expression pattern and coexpression networks of autism risk genes. a Violin plots showing the epxression levels of the nine hcASD genes in each neuron sub-cluster. $\mathbf{b}$ Enrichment analysis of the hcASD genes and pASD genes in each neuron sub-cluster and each region of the Ex 4 with neuron cell number over 5. c Coexpression network showing the top 1,000 genes coexpressed with the nine hcASD genes. This indicates more candidate ASD genes. d Enriched biological processes for genes coexpressed with the nine hcASD genes. e The expression network for the hcASD genes and the PASD genes

Cajal-Retzius cells and oligodendrocytes, expressed more genes and contained more abundant transcripts in each individual cell (Supplementary information, Figure S5). Similarly, the gene and transcript numbers increased along with the maturation of excitatory neurons. This effect was likely due to the increased transcription activity during the maturation of excitatory neurons (Fig. 2e and Supplementary information, Figure S2).

Spatial differences for different types of cells in the developing human cortex

The spatial position recorded for each cell provided valuable information for investigating the regional differences in the embryonic cerebral cortex in terms of regional cell type diversity. The four main regions in the cortex, the frontal lobe, the parietal lobe, the occipital lobe, and the temporal lobe, consisted of more neurons (Fig. 7a), whereas regions in the inferior surface contained many more glial cells. We particularly observed a high abundance of astrocytes in the pons (Fig. 7a and Supplementary information, Figure S6a). Immunostaining for GFAP to detect cells from the pons, the IT and PC regions showed the most astrocytes in the pons. Impressively, the astrocytes in the pons showed more mature morphology, with longer processes than those in the PC and IT regions (Fig. 7b). To determine whether there was a distribution difference between the two sub-clusters of astrocytes, we performed in situ hybridization for RAMP3 (marking the Astro_1 sub-cluster) and PTGDS (marking the Astro_2 sub-cluster) on the pons and cortical regions. Both sub-clusters were much more abundant in the pons than those in the PC and IT (Fig. 7c). We also noticed more excitatory neurons in regions belonging to the frontal lobe and more inhibitory neurons in the regions belonging to the temporal lobe (Fig. 7a and Supplementary information, Figure S6a). To validate the distribution differences of 
740 the cerebral cortex, we did RT-qPCR to compare the expression levels of NEUROD2 and GAD1 in the PC and IT regions. In agreement with the sequencing data, the $P C$ region showed higher expression of NEUROD2, whereas the IT region showed higher expression of GAD1 (Supplementary information, Figure $\mathrm{S} 6 \mathrm{~b})$. Moreover, the immunostaining of NEUROD2 in PC and IT regions also showed relatively more excitatory neurons in the PC (Supplementary information, Figure S6c).

We next questioned whether there were differences in cell heterogeneity among different regions. We extracted regions with over 50 single cells analyzed by RNA-seq for specific neuron subclusters and calculated the correlation coefficients between individual cells within each region. The gene expression heterogeneity of both excitatory and inhibitory neurons in the frontal lobe was significantly lower than that of other regions for each neuron sub-cluster (Supplementary information, Figure S6d). In contrast, the excitatory neuron sub-clusters in each region were more homogeneous than the inhibitory sub-clusters in the same region, which was compatible with the rich diversity of interneurons in mature human cortex and mouse cerebral cortex. ${ }^{8,}$ 9,11

We next analyzed DEGs among the cortical regions based on different neural sub-clusters. For inhibitory neurons (Fig. 7d), we found that the GABA receptor GABRA2 showed region-specific expression. The neuronal functional genes MGLL, TRAP1, and $N L G N 2^{43-45}$ were more specifically expressed in the PC, indicating that regional DEGs may be involved in the formation of regionspecific functions of the corresponding regions. We also found that Ex_1/2 neurons played a special role in the caudal middle frontal lobe (CMF) (Fig. 7e). Neuronal progenitors in this region highly expressed several genes related to neural diseases. For example, CLU and CST3 are related to Alzheimer's disease. ${ }^{46,47}$ Other region-specific genes such as NTRK2, which was highly detected in PC and supra-maginal regions, was reported to regulate the survival of neurons, and its down-regulation may cause autism. ${ }^{48}$ The BDNF/TrkB (TrkB also known as NTRK2) signaling pathway was also reported to be important in memory and learning during neural development. ${ }^{49}$ NRGN showed regionally differential expressions in both inhibitory and mature excitatory neurons but barely detectable expression in the Ex_1/2 neurons (Fig. 7d, $f$ and Supplementary information, Figure S6e). RT-qPCR showed enriched expression of NRGN in the IG region, consistent with the single-cell RNA-seq data (Fig. 7g). Unexpectedly, we identified several myocardial genes, such as MYL7, TNNT2, and ACTC1, which were expressed in the ST (Fig. 7f). We observed that TNNT2 co-localized with the neural marker NEUROD2 in individual cells in both the $22 \mathrm{~W}$ and $23 \mathrm{~W}$ ST regions at the protein level (Fig. 7h and Supplementary information, Figure S6f). As a control, we did not find this group of cells in the pars orbitalis region (PAO) of the $22 \mathrm{~W}$ and $23 \mathrm{~W}$ embryos.

\section{Expression pattern and co-expression networks of autism risk genes}

Autism risk genes might be up-regulated in mid-gestation human cortical projection neurons. ${ }^{5,51}$ As we investigated the transcriptome of the whole cortex at this gestation stage at single-cell resolution, we further analyzed the expression patterns of the high-confidence autism spectrum disorder (hcASD) genes and probable ASD (pASD) genes. ${ }^{50}$ All 9 hcASD genes were detected in the neuronal sub-clusters (Fig. 8a), whereas both hcASD and pASD genes showed expression enrichment in the Ex_4 sub-cluster (Fig. 8b). This finding agreed with previous results suggesting that cortical projection neurons were related to the pathogenesis of autism. We then measured the expression of both sets of ASD genes in Ex_4 in different regions. The pASD genes showed accordant expression across all regions, whereas the hcASD genes showed much lower enrichment scores in the IP and IT regions than those in other regions (Fig. 8b). The co-expression network of these nine hCASD genes revealed a key role for ANK2 in the expression network, and no interaction of KATNAL2 with other hcASD genes (Fig. 8c and Supplementary information, Table S5). GO analysis of the co-expressing genes showed the most significant enrichment in keratinization. Other enriched biological processes were revealed, such as spinal cord motor neuron differentiation and epidermis morphogenesis (Fig. 8d). Interaction analysis of the hcASD genes with the PASD genes revealed abundant interactions between ANK2, SCN2A, and POGZ and the pASD genes (Fig. 8e). Both the expression patterns and networks indicated that multiple distinct pathways and processes were potentially involved in the ASD phenotype. In addition to the hcASD and pASD genes, there were more network genes participating in functions that might increase the risk for ASD when they went wrong.

\section{DISCUSSION}

In this study, we uncovered 29 cell sub-clusters within the midgestation stage of human embryonic cerebral cortex in vivo and identified the unique signature of each type of cell related to their biological functions, such as in the cell cycle, TF networks, and metabolism. Inhibitory neurons originating from the MGE and the CGE exhibited clear differences in both their gene expression signatures and distribution patterns. The $\mathrm{LHX}^{+}$inhibitory neurons tended to be enriched in the top dorsal regions of the cerebral cortex, whereas the $C A L B 2^{+}$ones were in the inferior regions. Our findings indicate the potential in vivo molecular regulation of the maturation process of human excitatory neurons, which provides additional clues to aid in the generation of neurons in vitro with specific physiological functions.

By comparing our single-cell data to those of previous studies, we observed drastic differences between embryonic neurons and adult neurons. The results also indicate that the embryonic excitatory neurons had different gene regulation networks during maturation compared to the inhibitory neurons, and the latter showed delayed maturation compared with the former. For the $S S T^{+}$inhibitory neurons, they are the earliest to show up as a subgroup during embryonic development. However, these $\mathrm{SST}^{+}$ neurons are still different from the adult ones as the former showed no maturity apart from the expression of SST. It is a very important question of why these $\mathrm{SST}^{+}$inhibitory neurons show up earlier than other subtypes. Previous studies proved a key role of inhibitory neurons in circuit development, ${ }^{52-55}$ and thus $S S T^{+}$ inhibitory neurons might play a vanguard role in constructing the local circuit. As different subtypes of inhibitory neurons have different impacts on circuits, for example, the efferents of SST neurons are to principal cells, whereas the efferents of vasointestinal peptide (VIP) interneurons are mainly to SST neurons, ${ }^{33}$ which in turn lead to a disinhibition of those principal cells. ${ }^{56-58}$ It is easy to understand that the VIP neurons are generated later than SST neruons during development as they need local cues from both exicitatory and inhibitory signals for their final positioning ${ }^{59,60}$ and morphological development, ${ }^{61}$ thus SST neurons should show up earlier in the cerebral cortex. Therefore, we infer that the early appearance of $\mathrm{SST}^{+}$inhibitory neurons at embryonic stage is not to inhibit functions of other neurons as they do in the adult circuits, but these $S S T^{+}$neurons are helping to form neural circuits. This hypothesis needs more experimental validation in the future.

Regional differences were observed at different aspects. First of all, different cortex regions showed different cell type constitutions, for example, the regions in the inferior surface contain more glia cells, whereas the cortical regions contain more neurons. Moreover, both the excitatory and inhibitory neurons showed asymmetric distributions among the cortical regions. Regions in the frontal lobe contain more excitatory neurons, whereas regions in the temporal lobe contain more inhibitory ones. These differences in cell type constitution could possibly lead to regional 
specificity of the neural networks and their functions. Besides, neurons of the same sub-cluster showed different degrees of heterogeneity within each region of the cortex, and the frontal lobe was the most homogeneous region for a specific neuron subcluster. The different degrees of heterogeneity might be related to the complex neural connections when neurons are fully developed. Moreover, we observed that the maturation of different cortex regions was not synchronized, which may reflect the facts of regional difference in development. At last, we found that even for the same type of neurons, regional specific gene expression patterns existed, and these DEGs were strongly related to known neural diseases or region-specific neural functions.

Neural disorders are affecting numerous patients' health and life and they are still urgent issues currently without effective solutions. Many of the neural disorders are proved to be connected to the neural developmental stages, ${ }^{62-66}$ and especially the autism spectrum disorder (ASD) was reported to be closely related to the neuron projection at mid-fetal stage in human. ${ }^{47}$ We further analyzed the ASD risk genes in each neuron sub-cluster and brain region, and their network genes were revealed, offering a more precise view of the disease genes' regulation in the midgestation stage of the human cerebral cortex.

In summary, our results contribute to understanding the regionalization of the human cerebral cortex, which lays the ground for dissecting molecular and cellular mechanisms of brain development and diseases.

\section{MATERIALS AND METHODS}

Human embryonic brain collection and dissection

Human embryonic brains were obtained from the Third Hospital of Peking University with agreement of the donors. The $22 \mathrm{~W}$ and $23 \mathrm{~W}$ brains were collected from twins, and we collected each female cerebral cortex from the right hemisphere. We collected the right brain cortex from a $22 \mathrm{~W}$ male embryo. Only a few cells were collected from the male cerebral cortex of the right brain at $22 \mathrm{~W}$. For dissection of cortical regions (Fig. 1a and Supplementary information, Table S1), we referred to The Human Brain during the Second Trimester, by Shirley A. Bayer. ${ }^{67}$ For each female right brain, we dissected 19 regions from the cerebral cortex, and we also collected the insular gyrus, the pons and the medulla from the inferior surface. We barely cut the germinal zone in each region.

Tissue digestion and single-cell RNA-seq library preparation The dissected regions were transferred into $500 \mu$ l hibernate $E$ medium (Invitrogen, Cat\# A1247601) with $2 \mathrm{mg} / \mathrm{ml}$ collagenase IV (Gibco, Cat\# 17104-019) and $20 \mathrm{U} / \mu \mathrm{l}$ DNase I (NEB, Cat\# M0303L). The samples were roughly pipetted to break down the tissue and then added to $500 \mu \mathrm{l}$ hibernate $\mathrm{E}$ medium containing $1 \mathrm{mg} / \mathrm{ml}$ papain (Sigma, Cat\# P4762) and $20 \mathrm{U} / \mu \mathrm{l}$ DNase I. The tissue fragments were kept at $37^{\circ} \mathrm{C}$ in a thermocycler for $5 \mathrm{~min}$. The sample was then pipetted thoroughly to obtain single cells. To stop digestion, the tubes were centrifuged at $300 \times g$ for $2 \mathrm{~min}$, the cell pellet was collected, the supernatant was discarded, and the cells were resuspended with $1 \mathrm{ml}$ hibernate $\mathrm{E}$ medium. We removed the block mass using a $40-\mu \mathrm{m}$ filter. The cell suspension was kept on ice to prevent cell death during the single-cell collection procedure. We randomly picked single cells that appeared to be alive and placed them in $2.5 \mu \mathrm{l}$ cell lysis buffer using a mouth pipet under a microscope. We modified the STRTseq method for amplification of single-cell transcriptomes by changing the reverse transcription primer, the induced cell barcode, and the unique molecular identifier (UMI). The final primer concentration for reverse transcription was $300 \mathrm{nM}$. The amplification primers at the $3^{\prime}$ end were chemically modified with biotin. Before library construction, we enriched the fragments containing the cell barcode and UMI with streptavidin beads. The cDNAs of all 96 cells with different cell barcodes were pooled together for one library construction. ${ }^{68}$ Each single cell was 741 sequenced for $2 \times 10^{6}$ of 150 -bp paired-end reads using an Illumina HiSeq 4000.

Processing of single-cell RNA-Seq data

Raw reads were first segregated based on the cell-specific barcode information in read 2 of the pair-ended reads. Then, sequences in read 1 were trimmed with customized scripts to remove the TSO sequence, the polyA tail sequence and sequences with low-quality bases $(\mathrm{N}>10 \%)$ or contaminated with adapters. Subsequently, the stripped read 1 sequences were aligned to the hg19 human reference genome (UCSC) using TopHat (version 2.0.12). ${ }^{69}$ Uniquely mapped reads were counted using htseq-count from the HTSeq package ${ }^{70}$ and then grouped based on the cell-specific barcodes. For each gene, we discarded duplicated transcripts with identical UMIs. Finally, the transcript number for each gene in each cell was quantified by the number of distinct UMls of that gene.

In total, we sequenced 4,664 single cells, and cells with fewer than 1,000 detected genes, 20,000 detected transcripts and 20\% mapping ratio were removed. We also removed cells with too many raw reads, as these cells may not truly be single cells, thus leaving 4,233 cells for further analysis. Because most of our single cells did not reach one million UMls, we normalized the expression value by $\log _{2}($ TPM $/ 10+1)$ rather than $\log _{2}($ TPM +1$)$ (TPM: transcripts per million). By doing so, we avoided counting each transcript several times and overestimating the gene expressions.

Nonlinear dimensional reduction (t-SNE) and clustering We visualized our 4,233 single cells by t-SNE using the Barnes-Hut algorithm (implemented in the Rtsne package in R). First, we used the Seurat method to select the highly variable genes (HVGs) based on the $\log _{2}(T P M / 10+1)$ expression values. Only genes with an expression level $>1$ and expressed in at least 3 single cells were considered, whereas single cells with $<1,000$ expressed genes were excluded, leaving 4,213 cells for the subsequent analysis. HVGs with an average expression greater than 1 and a dispersion greater than 1 were used as inputs for the t-SNE analysis.

To cluster the cells, we first used the Seurat Find Clusters function, which is an implementation of a graph-based clustering approach, to obtain clusters based on all the HVGs (for details, see http://satijalab.org/seurat/pbmc-tutorial.html). We merged the obtained clusters into three main groups, namely, a neuron group, a glial cell group, and a non-neural cell group (see Supplementary information, Figure S1a). Second, as the heterogeneity of fetal brain cells was limited, to further obtain accurate subgroups in each main group, we employed previously reported methods. ${ }^{26}$ For each iteration, this method can split a certain group into 2 subgroups. We could then decide whether the obtained subgroups should be separated into smaller subgroups based on the feature genes selected by the random forest algorithm and the DEGs of the obtained subgroups. Specifically, subgroups were classified and verified using unsupervised hierarchical clustering and a random forest algorithm in R. In brief, (1) we first calculated the gene expression variation for each gene across all cells as $\mathrm{CV}^{2}=$ variance/ mean $^{2}$, which was then fitted to an inverse distribution, and we chose genes with a CV $\mathrm{CV}^{2}$ beyond one standard error of the mean; (2) we then carried out hierarchical clustering and determined two clusters at the first split; (3) we performed a 10-fold random forest feature selection to select DEGs dividing the two clusters; (4) for each class, we selected samples with internal vote probabilities $>0.6$ as the training set to achieve an optimal classifier, which was used to predict the rest of the samples; (5) 100 runs of 10-fold random forest cross-validation (CV) were carried out, and the samples with internal vote probabilities $<0.55$ were abandoned; (6) we repeated steps $1-5$ on the newly formed classes to obtain finer clusters. Finally, 13 clusters, including 8 inhibitory neuron clusters, 4 excitatory neuron clusters, and 1 Cajal-Retzius cell cluster, were identified for the neuron group (see Figs. 1a and 2a), 6 clusters for 
742 the glial cell group (see Supplementary information, Figure S3a) and 10 clusters for the non-neural cell group (see Supplementary information, Figure S4a). In addition, subsequent t-SNE plots for each primary group were generated by the Rtsne package using the DEGs (see below) of all the clusters in that group, except in the neuron group, for which we did not include the Cajal-Retzius cell cluster.

Identification of DEGs and GO analysis

To identify unique cluster-specific marker genes, we used the Seurat

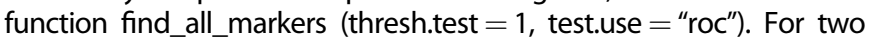
given clusters, the find.markers function was used to identify DEGs with the parameters thresh.use $=1$, test.use $=$ "roc". In the roc test, a value representing the 'classification power' ranging from 0 (for 'random') to 1 (for 'perfect') would be generated for a certain gene. We chose genes with a fold-change $\geq 2$ or $\leq 0.5$ and a power $\geq 0.4$ as DEGs. The R software program was used to plot heatmaps, pie charts, and bar plots. Violin plots were generated using Seurat. Enrichment analysis was performed using Metascape (http://metascape.org). ${ }^{71}$ Gene information involved in certain pathways was selected from KEGG (http://www.genome.jp/kegg/pathway.html). ${ }^{72}$

Developmental pseudotime analysis

The monocle2 package in $\mathrm{R}^{73,74}$ was used to determine the developmental pseudotime of excitatory neurons and glial cells. Following the monocle vignette, we used UMI count data as input and selected DEGs identified in the last procedure to order the cells. For all other parameters, the default settings were used. We also plotted eight TFs in DEGs along the inferred developmental pseudotime. The regulation networks for the 8 TFs were constructed by GENIE3 package and plotted by cytoscape.

For excitatory neurons, in Fig. 2e and Supplementary information, Figure S2 (right), we compared our dataset with two 21 pcw datasets from Miller et al. ${ }^{20}$ to see in which regions the subtype marker genes were enriched. We downloaded the microarray datasets and extracted the intersection genes with the genes identified to show regulation in excitatory neuron differentiation by monocle2. We then plotted heatmaps using $z$-score values with genes ordered as in Fig. 2e (left) and samples ordered from inner to outer layers of the prenatal neocortex.

\section{Cell cycle analysis}

A previously reported core gene set was used to perform cell cycle analyses, including $43 \mathrm{G} 1 / \mathrm{S}$ and $54 \mathrm{G} 2 / \mathrm{M}$ genes. ${ }^{75,76}$ We calculated the average expression of each gene set as the corresponding score. Cells were determined to be quiescent if their G1/S score $<2$ and G2/M score < 2; otherwise, they were deemed proliferative. In addition, proliferative cells were designated G2/ $M$ if their $\mathrm{G} 2 / \mathrm{M}$ score $>\mathrm{G} 1 / \mathrm{S}$ score, whereas cells were designated $\mathrm{G} 1 / \mathrm{S}$ if their G1/S score > G2/M score.

$\mathrm{LHXG}^{+}$interneuron and $C A L B 2^{+}$interneuron ratio in each specified cerebral cortex region

For each region, we defined the interneuron cells with a $\log _{2}$ (TPM of $L H X 6 / 10+1)$ greater than 1 as $\mathrm{LHXG}^{+}$interneurons. Similarly, interneuron cells with a $\log _{2}$ (TPM of CALB2/10 +1 ) greater than 1 were defined as $C A L B 2^{+}$interneurons. Then, the $L H X \sigma^{+}$interneuron ratio was calculated as the number of $L H X \sigma^{+}$interneurons to the total number of $\mathrm{LHX \sigma}^{+}$interneurons and $C A L B 2^{+}$interneurons. In addition, $\mathrm{LHXG}^{+}$regions were recognized as regions with a $\mathrm{LHX6^{+ }}$ interneuron ratio $>0.6 . C A L B 2^{+}$regions were identified in the same way. Regions that contained each type of interneuron between 0.4 and 0.6 were considered balanced regions. We did not include regions with a sum of both types of neurons less than 15 .

\section{Comparison with previous published datasets}

To reveal more developmental clues, we compared our dataset with three published datasets of the human cortex: two fetal ${ }^{11,27}$ and one adult dataset. ${ }^{26}$ We combined our dataset with the two fetal datasets using the mnnCorrect function of the scran package in $R^{37,38}$ This strategy relied on a shared subset of the population between batches and detected MNNs in the high-dimensional expression space to correct batch effects. The corrected dataset was then used to perform PCA through the FactoMineR package in R. Due to the abundance of cells in our dataset, there would have been PCA bias if we had used all the cells. Thus, we randomly selected 200 excitatory and 200 interneuron cells to redo the PCA. For each of the first four PC dimensions, the top 50 correlated genes combining both positively and negatively correlated genes were selected. We then performed unsupervised hierarchical clustering using Pearson correlation based on these genes through hclust in R. Clusterspecific markers were identified by $t$-test, and the $P$-value was corrected by the $\mathrm{BH}$ method using the p.adjust function.

For comparison with the adult cortex dataset, we first used Seurat to identify HVGs with an average expression $>1$ and dispersion $>1$ for both the adult dataset and our dataset separately. The expression value of the adult dataset was normalized by $\log _{2}($ TPM +1$)$ as used in the paper that it came from, while the expression value of our dataset was normalized by $\log _{2}(T P M / 10+1)$. Then, we calculated the $z$-score values for both datasets based on their own HVGs. Finally, we combined the two z-score-based datasets using all the HVGs to perform PCA through FactoMineR package in R. For each of the first four PCs, genes with correlation $>0.3$ or $<-0.3$ were selected to plot heatmaps and to perform enrichment analysis. However, for the PC1 positive direction, we chose the top 100 correlated genes because there were too many genes whose correlation exceeded 0.3 .

\section{Enrichment score for autism gene sets and gene regulation} network

To study the expression pattern of ASD-related genes, we collected two datasets described in Willsey et al.: 9 highconfidence ASD (hcASD) genes and 122 probable ASD (pASD) genes. ${ }^{50}$ We calculated the enrichment score for these two gene sets across all neuron cells using the AUCell package (https:// github.com/aertslab/GENIE3). ${ }^{77,78}$ Regulation networks for the 9 hcASD genes were constructed by the GENIE3 package (https:// github.com/aertslab/AUCell $)^{45}$ and plotted by Cytoscape.

\section{Regional heterogeneity of neurons across the whole cerebral}

cortex

For frontal, parietal, temporal, and occipital lobes, and inferior regions, we explored the regional heterogeneity of neurons by calculating the Pearson correlation between single cells of the heterogeneous genes for each sub-cluster whose cell number was $>200$. For each sub-cluster, we used Seurat to identify HVGs with an average expression $>1$ and a dispersion $>1$. We then calculated the Pearson correlation using these HVGs. For cells of each sub-cluster in a certain region, we first obtained the correlation distribution of single cells in that region, excluded self-correlation values (for which the value should be 1) and then calculated the correlation with cells in each of the other four regions. Wilcox.test in $\mathrm{R}$ was performed to test the significance of correlation between two distributions. To ensure that the conclusion was valid, we also identified HVGs with an average expression $>1$ and dispersion $>0.5$ to calculate the Pearson correlation and tested the significance of correlations between two distributions. The results of this procedure were similar (data not shown).

Developmental maturation degree across the whole cortex We used two independent methods to estimate the developmental maturation degree across the whole cortex. The first method evaluated synapse formation and function across cortical regions. The rationale was that a region is believed to be more mature if there are more cells that have formed synapses or whose 
synapses are functional. Thus, we calculated the mean expression of the gene set of GO terms 0007268-0007271 and 00160790016082 for each neuron (including both excitatory and inhibitory neurons) and averaged the expression values for a certain region, which was regarded as an indicator of synapse formation and function for that region. In addition, regions with fewer than 50 excitatory neurons were excluded. We then displayed the values of the cortical regions using heatmaps (see Fig. 4e).

The second method estimated the ratio of excitatory neurons expressing CUX2. Because CUX2 is an upper layer marker and cortical development follows an inside-out order for layer formation, a region is more mature if there are more excitatory neurons expressing CUX2. In this analysis, only excitatory neurons whose CUX2 expression value based on the $\log _{2}(T P M / 10+1)$ was $>1$ were deemed to exhibit true expression. Thus, for a certain region, the ratio of excitatory neurons expressing CUX2 was calculated as excitatory neurons expressing CUX2/all excitatory neurons. In addition, regions with fewer than 50 excitatory neurons were excluded. We also displayed these cortical region values using heatmaps (see Fig. 4c).

\section{RT-qPCR validation}

We dissected the IG, IT, PC, SP regions when we received the brain sample of a $22 \mathrm{~W}$ female embryo. Each region was digested into cell suspensions before extracting the total RNA with QIAGEN RNeasy mini kit (QIAGEN, Cat\# 74104). Then reverse transcription was carried out in a volume of $19 \mu \mathrm{l}$, containing $2.5 \mathrm{ng} / \mu \mathrm{l}$ oligod(T) primer (Takara, Cat\# 3806), $0.5 \mathrm{mM}$ dNTP mixture (Takara, Cat\# R045B), $2 \mathrm{U} / \mu \mathrm{l}$ RNase inhibitor (Ambion, Cat\# AM2684), $5 \mathrm{mM}$ DT,, $1 \times$ first strand and $0.5 \mathrm{U} / \mu \mathrm{l}$ superscript III reverse transcriptase (Invitrogen, Cat\# 18080-044). After reverse transcription at $50^{\circ} \mathrm{C}$ for $60 \mathrm{~min}$ and $70^{\circ} \mathrm{C}$ for $15 \mathrm{~min}$ to inactivate the enzyme, $1 \mu \mathrm{l}$ RNase H (Invitrogen, Cat\# 18021-071) was added into each reaction tube to digest RNA. For each sample we added nucleasefree water to obtain $1 \mathrm{ng} / \mu \mathrm{l}$ cDNA. The qPCR detection was run on CFX connect system (Biorad) using SYBR Green (Roche, Cat\# 13396700). Primers for each genes: GAPDH forward, CGACACCCACTCCTCCACCT, reverse, CTTGTGCTCTTGCTGGGGCT; NRGN forward, AGCGTCACCCAAGCACACTC, reverse, GCAAGGGTCGTCCG AAACCA; GAD1 forward, CGCTCTCTGTCTGGCTGTACG, reverse, ACAGTTGTGAGCCTGGTCACTT; NEUROD2 forward, GGTTCCCCC AAAAAGGGGCA, reverse, GGGTGTCCGACGGGAGTIC.

Immunohistochemistry

We fixed tissue samples in $4 \%$ paraformaldehyde for $16 \mathrm{~h}$, cryoprotected them in $30 \%$ sucrose, and embedded them in optimal cutting temperature compound (Thermo Scientific). Then, 40- $\mu \mathrm{m}$ cryosections were collected on Superfrost slides (VWR) using a Leica CM3050S cryostat. Primary antibodies rabbit antiNEUROD2 (1:500, Abcam ab104430), mouse anti-TNNT2 (1:100, Abcam ab8295), rabbit anti-GFAP (1:250, Sigma G9269) were diluted in blocking buffer containing $10 \%$ donkey serum, $0.5 \%$ Triton $\mathrm{X}-100$ and $0.2 \%$ gelatin diluted in PBS at $\mathrm{pH}$ 7.4. Binding was revealed using an appropriate Alexa FluorTM 488, Alexa FluorTM 594, or Alexa FluorTM 647 fluorophore-conjugated secondary antibody (Life Technologies). Cell nuclei were counter-stained using DAPI (Life Technologies). Images were collected using an Olympus FV1000 confocal microscope.

In situ hybridization

Probes complementary to target human mRNAs used for RNA in situ hybridization were cloned from primary human fetal cortical cDNA reverse-transcribed using PrimeScript II 1st Strand cDNA Synthesis Kit (Takara) with oligo dT primers. The RNA samples were isolated from $22 \mathrm{~W}$ and $23 \mathrm{~W}$ human cortex using SV Total RNA Isolation System (Promega). Specific genes were amplified using the following primers: RAMP3 forward, AAG GCT TTC GCA GAC ATG AT, reverse, ACA GGA TGC AGC AGG TGA TT;
Single-cell RNA-seq of human embryonic cerebral cortex $\mathrm{X}$. Fan et al.

PTGDS forward, GCT CCT CCT GCA CAC CTC, reverse, CAA TGG TAT 743 CCT CTG TGA AGC CC; CUX2 forward, CTG GAG AAG AAA GCC TAC $C T$, reverse, GAC AGG TGA CAC AGA CAT CAT G. Primers specific to target genes of interest were designed using Primer3. PCR used Q5 High-Fidelity DNA Polymerase (NEB). PCR products of predicted band sizes were gel-extracted and ligated using the Hieff CloneTM Plus One Step Cloning Kit (Yeason). Ligation products were transfected into Trans5a Chemically Competent $E$. coli (Transgene). Cloned sequences were confirmed by sequencing. Digoxigenin-labeled RNA probes for in situ hybridization were generated by linearizing the PSPT18 Vector and in vitrotranscribing the probe using T7 or SP6 RNA Polymerase (Roche) in the presence of DIG-RNA Labeling Mix (Roche). In situ hybridization was performed blinded to the sense/antisense status of each probe, and sense control probes gave no signal (data not shown). The in situ hybridization protocol was described previously (Inma Cobos).

Data and software availability

The accession number for all sequencing data reported in this paper is GEO: GSE103723 (The following secure token has been created to allow review of record GSE103723 while it remains in private status: wnyxaammzloppsl). The expression pattern of our single-cell RNA-seq data are available from the corresponding author upon request.

\section{ACKNOWLEDGEMENTS}

This study was supported by the National Natural Science Foundation of China (31625018, 81561138005, 31230047, 81521002 and 91732301), the National Basic Research Program of China (2014CB964600 and 2017YFA0103303), the Strategic Priority Research Program of the Chinese Academy of Sciences (XDA16020601), Shanghai Brain-Intelligence Project from STCSM (16JC1420500), and the Beijing Advanced Innovation Center for Genomics at Peking University. High-throughput sequencing was performed in Novogene.

\section{AUTHOR CONTRIBUTIONS}

T.F., Q.J. and W.X.Q. designed the project. F.X., Z.S. and W.Y. carried out most of the experiments; D.J. did the bioinformatic analysis; W.X.Y., Y.J. and W.W. contributed to the brain sample collection; S.L., Y.L., Z.Y. and Y.J. helped with the immunostaining for data validation. F.X., D.J. and W.Q. interpreted the results and wrote the manuscript: T.F. and W.X.Q. led the experimental design and revised the manuscript.

\section{ADDITIONAL INFORMATION}

Supplementary information accompanies this paper at https://doi.org/10.1038/ s41422-018-0053-3.

Competing interests: The authors declare no competing interests.

\section{REFERENCES}

1. McKay, R. D. G. The origins of cellular diversity in the mammalian central nervous system. Cell 58, 815-821 (1989).

2. Masland, R. H. Neuronal cell types. Curr. Biol. 14, R497-R500 (2018),

3. Wright, I. C., Sham, P., Murray, R. M., Weinberger, D. R. \& Bullmore, E. T. Genetic contributions to regional variability in human brain structure: methods and preliminary results. Neuroimage 17, 256-271 (2002).

4. Shipp, S. Structure and function of the cerebral cortex. Curr. Biol. 17, 443-449 (2007).

5. Yoshimura, Y., Dantzker, J. L. M. \& Callaway, E. M. Excitatory cortical neurons form fine-scale functional networks. Nature 433, 868 (2005).

6. West, D. C., Mercer, A., Kirchhecker, S., Morris, O. T. \& Thomson, A. M. Layer 6 cortico-thalamic pyramidal cells preferentially innervate interneurons and generate facilitating EPSPs. Cereb. Cortex 16, 200-211 (2006).

7. Sillito, A. M., Cudeiro, J. \& Jones, H. E. Always returning: feedback and sensory processing in visual cortex and thalamus. Trends Neurosci. 29, 307-316 (2006).

8. Zeisel, A. et al. Cell types in the mouse cortex and hippocampus revealed by single-cell RNA-seq. Science 347, 1138-1142 (2015). 
744 9. Tasic, B. et al. Adult mouse cortical cell taxonomy revealed by single cell transcriptomics. Nat. Neurosci. 19, 335-346 (2016).

10. Zeng, H. et al. Large-scale cellular-resolution gene profiling in human neocortex reveals species-specific molecular signatures. Cell 149, 483-496 (2012).

11. Darmanis, S. et al. A survey of human brain transcriptome diversity at the single cell level. Proc. Natl Acad. Sci. USA 112, 7285-7290 (2015).

12. Poulin, J., Tasic, B., Hjerling-Leffler, J., Trimarchi, J. M. \& Awatramani, R. Disentangling neural cell diversity using single-cell transcriptomics. Nat. Neurosci. 19, 1131-41 (2016).

13. Costa, M. R. \& Müller, U. Specification of excitatory neurons in the developing cerebral cortex: progenitor diversity and environmental influences. Front. Cell Neurosci. 8, 449 (2014).

14. Brandão, J. A. \& Romcy-Pereira, R. N. Interplay of environmental signals and progenitor diversity on fate specification of cortical GABAergic neurons. Front. Cell. Neurosci. 9, 149 (2015).

15. Simons, M. \& Trajkovic, K. Neuron-glia communication in the control of oligodendrocyte function and myelin biogenesis. J. Cell Sci. 119, 4381 LP-4384389 (2006).

16. Schitine, C., Nogaroli, L., Costa, M. R. \& Hedin-Pereira, C. Astrocyte heterogeneity in the brain: from development to disease. Front. Cell Neurosci. 9, 76 (2015).

17. Parpura, V. et al. Glial cells in (patho)physiology. J. Neurochem. 121, 4-27 (2012).

18. Zuchero, J. B. \& Barres, B. A. Glia in mammalian development and disease. Development 142, 3805-3809 (2015).

19. Chou, S. et al. Analysis of spatial-temporal gene expression patterns reveals dynamics and regionalization in developing mouse brain. Sci. Rep. 6, 19274 (2016).

20. Miller, J. A. et al. Transcriptional landscape of the prenatal human brain. Nature 508, 199-206 (2014).

21. Bakken, T. E. et al. A comprehensive transcriptional map of primate brain development. Nature 535, 367-375 (2016).

22. Erclik, T. et al. Integration of temporal and spatial patterning generates neural diversity. Nature 541, 365-370 (2017).

23. Kang, H. J. et al. Spatiotemporal transcriptome of the human brain. Nature $\mathbf{4 7 8}$, 483 (2011).

24. Competition, L., Affects, D. \& In, I. The developmental transcriptome of the human brain: Implications for neurodevelopmental disorders. Curr. Opin. Neurol. 86, 3279-3288 (2008).

25. Usoskin, D. et al. Unbiased classification of sensory neuron types by large-scale single-cell RNA sequencing. Nat. Neurosci. 18, 145-153 (2015).

26. Lake, B. B. et al. Neuronal subtypes and diversity revealed by single-nucleus RNA sequencing of the human brain. Science 352, 1586-1590 (2016).

27. Pollen, A. A. et al. Low-coverage single-cell mRNA sequencing reveals cellular heterogeneity and activated signaling pathways in developing cerebral cortex. Nat. Biotechnol. 32, 1053-1058 (2014).

28. Johnson, M. B. et al. Single-cell analysis reveals transcriptional heterogeneity of neural progenitors in human cortex. Nat. Neurosci. 18, 637-646 (2015).

29. Zhong, S. et al. A single-cell RNA-seq survey of the developmental landscape of the human prefrontal cortex. Nature 555, 524-528 (2018).

30. Nowakowski, T. J. et al. Spatiotemporal gene expression trajectories reveal developmental hierarchies of the human cortex. Science 358, 1318-1323 (2017).

31. Li, C.-L. et al. Somatosensory neuron types identified by high-coverage single-cell RNA-sequencing and functional heterogeneity. Cell Res. 26, 83-102 (2015).

32. Herculano-Houzel, $\mathrm{S}$. The glia/neuron ratio: how it varies uniformly across brain structures and species and what that means for brain physiology and evolution. Glia 62, 1377-1391 (2014).

33. Kepecs, A. \& Fishell, G. Interneuron cell types are fit to function. Nature $\mathbf{5 0 5}$, 318-326 (2014).

34. Hansen, D. V. et al. Non-epithelial stem cells and cortical interneuron production in the human ganglionic eminences. Nat. Neurosci. 16, 1576-1587 (2013).

35. Machon, O., Masek, J., Machonova, O., Krauss, S. \& Kozmik, Z. Meis2 is essential for cranial and cardiac neural crest development. BMC Dev. Biol. 15, 40 (2015).

36. Goff, L. A. et al. Spatiotemporal expression and transcriptional perturbations by long noncoding RNAs in the mouse brain. Proc. Natl Acad. Sci. USA 112, 6855-6862 (2015).

37. Sauvageau, M. et al. Multiple knockout mouse models reveal lincRNAs are required for life and brain development. Elife 2, e01749 (2013).

38. Precious, S. V. et al. FoxP1 marks medium spiny neurons from precursors to maturity and is required for their differentiation. Exp. Neurol. 282, 9-18 (2016).

39. Zimmer, C., Tiveron, M. C., Bodmer, R. \& Cremer, H. Dynamics of Cux2 expression suggests that an early pool of SVZ precursors is fated to become upper cortical layer neurons. Cereb. Cortex 14, 1408-1420 (2004).

40. Franco, S. J. et al. Fate-restricted neural progenitors in the mammalian cerebral cortex. Science 337, 746-749 (2012).

41. Haghverdi, L., Lun, A. T. L., Morgan, M. D. \& Marioni, J. C. Batch effects in singlecell RNA-sequencing data are corrected by matching mutual nearest neighbors. Nat. Biotechnol. 36, 421-427 (2018).
42. L. Lun, A. T., Bach, K. \& Marioni, J. C. Pooling across cells to normalize single-cell RNA sequencing data with many zero counts. Genome Biol. 17, 75 (2016).

43. Amoroso, M. R. et al. TRAP1 downregulation in human ovarian cancer enhances invasion and epithelial-mesenchymal transition. Cell Death Dis. 7, e2522 (2016).

44. Babaev, O. et al. Neuroligin 2 deletion alters inhibitory synapse function and anxiety-associated neuronal activation in the amygdala. Neuropharmacology $\mathbf{1 0 0 ,}$ 56-65 (2016).

45. Chandley, M. J., Crawford, J. D., Szebeni, A., Szebeni, K. \& Ordway, G. A. NTRK2 expression levels are reduced in laser captured pyramidal neurons from the anterior cingulate cortex in males with autism spectrum disorder. Mol. Autism 6, 28 (2015).

46. Mi, W. et al. Cystatin C inhibits amyloid-beta deposition in Alzheimer's disease mouse models. Nat. Genet. 39, 1440-1442 (2007).

47. Braskie, M. N. et al. Common Alzheimer's disease risk variant within the CLU gene affects white matter microstructure in young adults. J. Neurosci. 31, 6764-6770 (2011).

48. Shamir, A. et al. The importance of the NRG-1/ErbB4 pathway for synaptic plasticity and behaviors associated with psychiatric disorders. J. Neurosci. 32, 2988-2997 (2012).

49. Minichiello, L. TrkB signalling pathways in LTP and learning. Nat. Rev. Neurosci. 10, 850-860 (2009).

50. Willsey, A. J. et al. Coexpression networks implicate human midfetal deep cortical projection neurons in the pathogenesis of autism. Cell 155, 997-1007 (2013).

51. State, M. W. \& Šestan, N. The emerging biology of autism spectrum disorders. Science 337, 1301-1303 (2012).

52. Hensch, T. K. et al. Local GABA circuit control of experience-dependent plasticity in developing visual cortex. Science 282, 1504-1508 (1998).

53. Richards, B. A., Voss, O. P. \& Akerman, C. J. GABAergic circuits control stimulusinstructed receptive field development in the optic tectum. Nat. Neurosci. 13, 1098-1106 (2010).

54. Gabernet, L., Jadhav, S. P., Feldman, D. E., Carandini, M. \& Scanziani, M. Somatosensory integration controlled by dynamic thalamocortical feed-forward inhibition. Neuron 48, 315-327 (2018).

55. Le Magueresse, C. \& Monyer, H. GABAergic interneurons shape the functional maturation of the cortex. Neuron 77, 388-405 (2013).

56. Pfeffer, C. K., Xue, M., He, M., Huang, Z. J. \& Scanziani, M. Inhibition of inhibition in visual cortex: The logic of connections between molecularly distinct interneurons. Nat. Neurosci. 16, 1068-1076 (2013).

57. Lee, S., Kruglikov, I., Huang, Z. J., Fishell, G. \& Rudy, B. A disinhibitory circuit mediates motor integration in the somatosensory cortex. Nat. Neurosci. 16, $1662-1670$ (2013).

58. $\mathrm{Pi}, \mathrm{H}$.-J. et al. Cortical interneurons that specialize in disinhibitory control. Nature 503, 521-524 (2013).

59. Cancedda, L., Fiumelli, H., Chen, K. \& Poo, M. Excitatory GABA action is essential for morphological maturation of cortical neurons in vivo. J. Neurosci. 27, 5224-5235 (2007).

60. Bortone, D. \& Polleux, F. KCC2 expression promotes the termination of cortical interneuron migration in a voltage-sensitive calcium-dependent manner. Neuron 62, 53-71 (2009).

61. De Marco García, N. V., Karayannis, T. \& Fishell, G. Neuronal activity is required for the development of specific cortical interneuron subtypes. Nature 472, 351-355 (2011).

62. Oliveira, M. A. P., Balling, R., Smidt, M. P. \& Fleming, R. M. T. Embryonic development of selectively vulnerable neurons in Parkinson's disease. NPJ Park. Dis. 3, 21 (2017).

63. Nasir, J. et al. Targeted disruption of the Huntington's disease gene results in embryonic lethality and behavioral and morphological changes in heterozygotes. Cell 81, 811-823 (2018).

64. Oppenheim, R. W. Cell death during development of the nervous system. Annu. Rev. Neurosci. 14, 453-501 (1991).

65. Heanue, T. A. \& Pachnis, V. Enteric nervous system development and Hirschsprung's disease: advances in genetic and stem cell studies. Nat. Rev. Neurosci. 8, 466-479 (2007).

66. Tebbenkamp, A. T. N., Willsey, A. J., State, M. W. \& Šestan, N. The developmental transcriptome of the human brain: implications for neurodevelopmental disorders. Curr. Opin. Neurol. 27, 149-156 (2014).

67. Bayer, S. A., Altman, J. (eds). The Human Brain During the Second Trimester. (CRC Press, Boca Raton, 2005).

68. Li, L. et al. Single-cell RNA-Seq analysis maps development of human germline cells and gonadal niche interactions. Cell Stem Cell 20, 858-873 (2017).

69. Trapnell, C., Pachter, L. \& Salzberg, S. L. TopHat: discovering splice junctions with RNA-Seq. Bioinformatics 25, 1105-1111 (2009).

70. Anders, S., Pyl, P. T. \& Huber, W. HTSeq-A Python framework to work with highthroughput sequencing data. Bioinformatics 31, 166-169 (2015). 
71. Tripathi, S. et al. Meta- and orthogonal integration of influenza 'OMICs' data defines a role for UBR4 in virus budding. Cell Host Microbe 18, 723-735 (2015).

72. Ogata, H. et al. KEGG: kyoto encyclopedia of genes and genomes. Nucleic Acids Res. 27, 29-34 (1999).

73. Trapnell, C. et al. The dynamics and regulators of cell fate decisions are revealed by pseudotemporal ordering of single cells. Nat. Biotechnol. 32, 381-386 (2014).

74. Qiu, X. et al. Single-cell mRNA quantification and differential analysis with Census. Nat. Methods 14, 309-315 (2017).

75. Tirosh, I. et al. Dissecting the multicellular ecosystem of metastatic melanoma by single-cell RNA-seq. Science 352, 189-196 (2016).

76. Macosko, E. Z. et al. Highly parallel genome-wide expression profiling of individual cells using nanoliter droplets. Cell 161, 1202-1214 (2015).

77. Huynh-Thu, V. A., Irrthum, A., Wehenkel, L. \& Geurts, P. Inferring regulatory networks from expression data using Tree-based methods. PLoS One 5, e12776 (2010).

78. Aibar, S. et al. SCENIC: single-cell regulatory network inference and clustering. Nat. Methods 14, 1083 (2017).
Single-cell RNA-seq of human embryonic cerebral cortex $X$. Fan et al.

(c) (i) Open Access This article is licensed under a Creative Commons Attribution 4.0 International License, which permits use, sharing, adaptation, distribution and reproduction in any medium or format, as long as you give appropriate credit to the original author(s) and the source, provide a link to the Creative Commons license, and indicate if changes were made. The images or other third party material in this article are included in the article's Creative Commons license, unless indicated otherwise in a credit line to the material. If material is not included in the article's Creative Commons license and your intended use is not permitted by statutory regulation or exceeds the permitted use, you will need to obtain permission directly from the copyright holder. To view a copy of this license, visit http://creativecommons. org/licenses/by/4.0/.

(c) The Author(s) 2018 